\title{
Unterschiede in der institutionellen Einbettung der Arbeitsbeziehungen und Human Resource Management Praktiken zwischen Deutschland und der Schweiz: eine explorative Untersuchung in Schweizer Tochtergesellschaften deutscher MNU
}
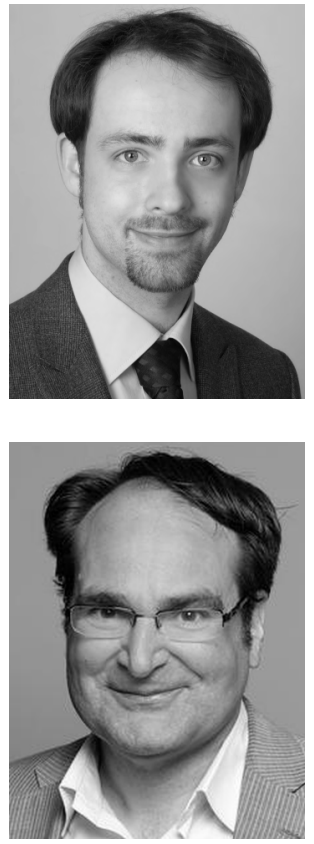

\section{Oliver Schröter, Eric Davoine}

Personalmanagement, Human Resource Management, Arbeitsmarkt, Arbeitsbeziehungen, industrielle Beziehungen, Deutschland, Schweiz

Personnel management, human resource management, labour market, employee relations, industrial relations, Germany, Switzerland

Der Beitrag untersucht Arbeitsbeziehungen und HRM Praktiken in der Schweiz aus der Perspektive von Schweizer Niederlassungen deutscher multinationaler Unternehmen (MNU). Die Schweiz und Deutschland sind zwei Länder, die neben zahlreichen Ähnlichkeiten auch bedeutende institutionelle Unterschiede aufweisen. Durch die Untersuchung des Transfers von HRM Praktiken in neun Fallstudien werden Zusammenhänge zwischen institutionellem Umfeld und organisationalen Praktiken hergestellt. Aus dieser Analyse geht hervor, dass wesentliche Merkmale wie stabile Beschäftigungsverhältnisse und Praktiken wie duale Berufsausbildung, Einbeziehung der Sozialpartner und Mitbestimmung am Arbeitsplatz in der Schweiz in sehr ähnlicher Weise vorzufinden sind wie in Deutschland. HRM Praktiken in der Schweiz werden aber durch teils sehr unterschiedliche institutionelle und gesellschaftliche Konstellationen bedingt.

The present contribution investigates HRM practices in Switzerland viewed from the perspective of Swiss subsidiaries of German multinational companies (MNCs). Switzerland and Germany are two countries that show many similarities but also important differences in their respective institutional settings. Through an investigation of HRM practice transfer in nine case studies, we identified interrelations between national institutions and organizational practices. The analysis shows that central characteristics like stable employment relations and practices like dual vocational education and training, inclusion of social partners and co-determination at the working place may exist in fairly similar ways as in Germany. Though, some HRM practices in Switzerland are conditioned by different elements of the local institutional and societal settings. 


\section{Einleitung: Originalität der Fragestellung und Beobachtungsperspektive}

Human Resource Management (HRM) in der Schweiz war bereits Gegenstand vergleichender Studien, in deren Rahmen das Land gemeinsam mit Österreich als kleine deutschsprachige Länder des Alpenraums behandelt (Erten et al. 2004) oder der Gruppe deutschsprachiger Länder zugeteilt wurde (Avery et al. 1999; Hofstede 2001). In der Varieties of Capitalism (VoC) Theorie werden Deutschland und die Schweiz gewöhnlich der selben Kategorie koordinierter Marktwirtschaften zugeordnet (Soskice 1999, 204; Hall/Gingerich 2004, 10; Hall/Gingerich 2009, 458, 479).

Während eine gemeinsame Betrachtung Deutschlands und der Schweiz vor dem Hintergrund gewisser sprachlicher und kultureller Gemeinsamkeiten sowie weiterer Ähnlichkeiten hinsichtlich wesentlicher Institutionen sinnvoll erscheint, so weisen doch die Studien von Schneider und Paunescu (2012) sowie Teuber und Backes-Gellner (2013) auf die Existenz bedeutender Unterschiede hin, die eine getrennte Betrachtung der Schweiz rechtfertigen. Der folgende Beitrag geht den Fragen nach, wo genau diese Unterschiede zwischen Deutschland und der Schweiz liegen, und ob diese Unterschiede zu nachweisbaren Konsequenzen für Arbeitsbeziehungen und HRM Praktiken führen.

Obgleich Schweizer Unternehmen international erfolgreich sind und die Schweiz im Global Competitiveness Report (Schwab/Sala-i-Martin 2012, 11, 13) wiederholt den ersten Platz einnimmt, so sind die HRM Praktiken dieses Landes noch vergleichsweise wenig aus der institutionellen Perspektive untersucht worden. Vorhandene Studien, die sich mit Schweizer Management und HRM Praktiken beschäftigen, sind mehrheitlich kulturalistisch (Weibler/Wunderer 2007) und oft bereits älteren Datums (Laurent 1985; Bergmann 1990; Bergmann 1994; Tixier 1994), oder dem Feld der international vergleichenden Managementforschung zuzuordnen (Morley et al. 2000; Erten et al. 2004).

Als kleines, stark internationalisiertes Land besitzt die Schweiz bestimmte eigene Charakteristika, die einen direkten Vergleich mit Deutschland methodisch problematisch machen. Dies spiegelt sich zum einen konkret in der im europäischen Vergleich auffällig internationalen Besetzung schweizerischer Unternehmensspitzen wider (Davoine 2005; Davoine/Ravasi 2013). Zum anderen erwirtschafteten ausländische und schweizerische multinationale Unternehmen (MNU) zusammen im Jahr 2004 etwa ein Drittel des Bruttoinlandsprodukts (Naville et al. 2007). In diesem Zusammenhang weist die Schweiz die weltweit höchste Anzahl an MNU pro 100000 Einwohnern auf (Erten et al. 2004, 102).

Angesichts dieser enormen lokalen Präsenz von MNU erscheint es naheliegend, HRM Praktiken in der Schweiz einmal nicht aus einer klassischen, rein vergleichenden oder kulturalistischen Beobachtungsperspektive zu erforschen, sondern anhand von Fallstudien, die in den Schweizer Niederlassungen ausländischer MNU durchgeführt werden.

Der Transfer von HRM Praktiken in Auslandsniederlassungen deutscher MNU bietet sich hierbei besonders an, um Unterschiede zwischen Deutschland und der Schweiz zu untersuchen. Denn die Denk- und Handelsweisen der Akteure im Gastland sind geprägt von unterschiedlichen „embedded managerial norms“ (Almond 2011, 265), wodurch es $\mathrm{zu}$ einer Kollision unterschiedlicher Typen institutioneller Rationalität kommt (Ferner et al. 2012, 166). In solchen Situationen treten Unterschiede also besonders deutlich zutage und können gut beobachtet werden. 


\section{Studien zum Transfer von HRM Praktiken in MNU}

In der einschlägigen Literatur zum internationalen Transfer von HRM Praktiken werden diverse Effekte unterschieden, die diesen Prozess und seine Ergebnisse wesentlich beeinflussen (Edwards/Ferner 2002; Almond et al. 2005; Edwards et al. 2007). Zunächst steht hier der Einfluss des Ursprungslandes oder der country-of-origin Effekt demjenigen des Gastlandes oder host country Effekt gegenüber. Diese Einflüsse sind zurückzuführen auf die Einbettung organisationaler Praktiken in ein jeweiliges Umfeld, wobei wirtschaftliches Handeln immer innerhalb bestimmter sozialer, kultureller, politischer und kognitiver Strukturen stattfindet und durch diese strukturiert wird (Granovetter 1985; Beckert 2003, 769).

Hier wiederum spielen Institutionen eine zentrale Rolle, da diese strategische Entscheidungen beeinflussen, indem sie den Rahmen dafür festlegen, was innerhalb eines gegebenen Kontextes möglich und vorteilhaft ist (Dacin et al. 1999, 324). Der country-of-origin Effekt spiegelt hierbei einen überproportionalen Einfluss des Ursprungslandes wieder, in dem Organisationen eingebettet sind. Dieser spezifische Einfluss lässt sich auch in stark internationalisierten MNU nachweisen und spiegelt sich in der Art wider, wie sich MNU internationalisieren und wie sie ihr Personal managen (Ferner 1997; Wächter et al. 2003). Host country Effekte (Ferner et al. 2001; Almond et al. 2005) andererseits ergeben sich aus den institutionellen Regulierungen des Gastlandes, die den Organisationen bei der Formulierung ihrer HRM Praktiken mehr oder weniger Freiraum gewähren können (Marginson et al. 1993; Ferner 1994).

$\mathrm{Zu}$ diesen beiden Faktoren kommen globale Dominanzeffekte (Smith/Meiksins 1995), die insbesondere auf die wirtschaftliche Vormachtstellung der USA und die Vorbildrolle US-amerikanischer MNU zurückzuführen sind (Djelic 1998). Ferner werden weitere Einflüsse mit einbezogen, die sich aus Struktur, Strategie und Integration der Aktivitäten der MNU (Müller 1994) sowie den Handlungsstrategien bzw. Machtressourcen diverser Akteure ergeben (Edwards/Rees 2006, 84; Dörrenbächer/Gammelgaard 2011; Ferner et al. 2012).

Für den vorliegenden Beitrag erscheint es jedoch sinnvoll, sich insbesondere auf deutsche country-of-origin sowie schweizerische host country Effekte zu konzentrieren, um auf diese Weise herauszuarbeiten, wo genau Unterschiede zwischen den beiden Ländern bestehen und welche Konsequenzen sich hieraus für das Human Resource Management und die industriellen Beziehungen Ergeben.

Bevor wir uns näher einer Analyse der Unterschiede zwischen dem deutschen und schweizerischen institutionellen Umfeld widmen, werden wir zunächst die wichtigsten Ergebnisse vorangegangener Studien zum Transfer von HRM Praktiken in deutschen MNUs vorstellen.

\subsection{Deutsche MNU und Transfer von HRM Praktiken in andere Gastländer}

Wie aus Tabelle 1 ersichtlich wird, ist der Transfer von Praktiken des HRM und der IB (industrielle Beziehungen) in deutschen MNU bislang hauptsächlich in institutionell gesehen relativ unterschiedlichen Gastländern wie Grossbritannien, Spanien oder mittel- bzw. osteuropäischen Transitionsländern untersucht worden. Wie wir eingangs festgestellt haben, liegen hierzu jedoch bislang keinerlei Erkenntnisse über die Schweiz als Gastland vor. 


\begin{tabular}{|c|c|c|}
\hline Gastland & Autoren und Jahr & Reaktion der deutschen MNU \\
\hline UK & $\begin{array}{l}\text { (Beaumont et al. } \\
\text { 1990; Guest/ } \\
\text { Hoque } 1996 ; \text { Fer- } \\
\text { ner/Varul 2000a; } \\
\text { Ferner et al. 2001; } \\
\text { Tempel 2001; Tü- } \\
\text { selmann et al. } \\
\text { 2001; Faulkner et } \\
\text { al. 2002; Dick- } \\
\text { mann 2003; Tü- } \\
\text { selmann et al. } \\
\text { 2003; Tüselmann } \\
\text { et al. 2006; } \\
\text { Marginson et al. } \\
\text { 2010) }\end{array}$ & 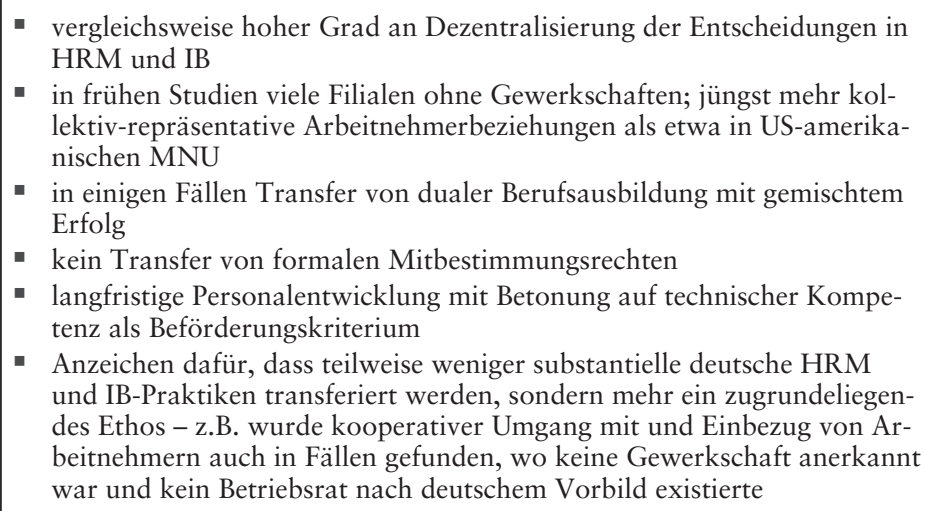 \\
\hline Spanien & $\begin{array}{l}\text { (Ferner et al. } \\
\text { 2001; Dickmann } \\
\text { 2003) }\end{array}$ & $\begin{array}{l}\text { Duale Berufsausbildung als bewährtes Mittel zur Personalselektion und/ } \\
\text { oder aufgrund wahrgenommenen Mangels adäquat ausgebildeter Ar- } \\
\text { beitskräfte auf lokalen Arbeitsmärkten transferiert } \\
\text { - Massnahmen zur aktiven Verbesserung der Arbeitsbeziehungen und zur } \\
\text { Vorbeugung von Arbeitskonflikten, die als Ausdruck eines typisch deut- } \\
\text { schen Ethos gedeutet wurden } \\
\text { - In Industriefirmen waren Arbeitnehmervertretungen und Gewerkschaf- } \\
\text { ten präsent, in Banken nicht }\end{array}$ \\
\hline Korea & $\begin{array}{l}\text { (Chang 2004; } \\
\text { Chang 2006) }\end{array}$ & $\begin{array}{l}\text { vergleichsweise hoher Grad an Dezentralisierung der Entscheidungen in } \\
\text { HRM und IB } \\
\text { - Kombination von leistungsabhängigem Entgelt mit traditionellen senio- } \\
\text { ritätsbasierten Systemen zu neuen Hybridpraktiken } \\
\text { - kein Transfer von dualer Berufsausbildung; dennoch starke Betonung } \\
\text { technischer Aus- und Weiterbildung, die teils in Deutschland durchge- } \\
\text { führt wurde } \\
\text { - kooperative IB mit Gewerkschaften und Kollektivverträge }\end{array}$ \\
\hline $\begin{array}{l}\text { Tschechische } \\
\text { Republik } \\
\text { und Polen }\end{array}$ & (Blubm 2001) & $\begin{array}{l}\text { - Schwierigkeiten bei Einführung flexibler Arbeitszeitmodelle aufgrund re- } \\
\text { striktiver Gesetzgebung und niedriger Löhne } \\
\text { - Kooperation mit lokalen Berufsschulen: Schaffung funktionaler Äquiva- } \\
\text { lente zu deutscher dualer Berufsausbildung } \\
\text { - MNU folgten eher deutschem Modell als KMU } \\
\text { - Beziehungen grosser MNU mit Gewerkschaften ähnelten denjenigen mit } \\
\text { deutschen Betriebsräten }\end{array}$ \\
\hline Ungarn & $\begin{array}{l}\text { (Dörrenbächer } \\
\text { 2004) }\end{array}$ & $\begin{array}{l}\text { - Selektiver oder kein Transfer von deutschem Modell der Arbeitsbezie- } \\
\text { hungen } \\
\text { - viele individualistische Elemente (individuelle Lohnverhandlungen) } \\
\text { - grosse Unternehmen fühlten sich eher sozialpartnerschaftlichen Grund- } \\
\text { sätzen verpflichtet als kleine }\end{array}$ \\
\hline $\begin{array}{l}\text { Polen, Un- } \\
\text { garn und } \\
\text { Slowenien }\end{array}$ & $\begin{array}{l}\text { (Meardi 2007; } \\
\text { Meardi et al. } \\
\text { 2009) }\end{array}$ & $\begin{array}{l}\text { - Kontrollpraktiken vom Hauptsitz, Teamwork und flexible Löhne als } \\
\text { "disembedded global best practices“ } \\
\text { - starke Betonung auf Aus- und Weiterbildung und technische Kompetenz } \\
\text { - Kooperation mit lokalen Schulen für Lehrlingsausbildung } \\
\text { - Jahresarbeitszeitkonten } \\
\text { - Unterschiede innerhalb derselben deutschen MNU zwischen polnischer } \\
\text { und ungarischer Niederlassung }\end{array}$ \\
\hline
\end{tabular}

Tabelle 1: Studien zum internationalen Transfer von HRM Praktiken in deutschen MNU (Quelle: eigene Darstellung) 
Fassen wir einige wesentliche Ergebnisse vorangegangener Studien zum Transfer von Praktiken im Bereich HRM und IB zusammen, so können wir festhalten, dass einige Tendenzen sichtbar werden.

Zunächst geht aus diversen Studien hervor, dass deutsche MNU im Bereich HRM und IB oftmals relativ stark dezentralisiert sind und diesbezügliche Entscheidungen entsprechend auf Ebene der einzelnen Auslandsfilialen getroffen werden (Guest/Hoque 1996, 64; Ferner/Varul 2000b, 89; Ferner/Varul 2000a, 132; Chang 2006). Zudem wurde festgestellt, dass deutsche MNU häufig weniger substantielle deutsche HRM und IB Praktiken als eine zugrundeliegende Philosophie, ein Ethos oder ein bestimmtes deutsches Mindset transferieren (Dickmann 2003, 279; Tüselmann et al. 2006, 69). Dies ist teils bedingt durch fehlende institutionelle Unterstützung oder Infrastrukturen im jeweiligen Gastland, die für einen vollen Transfer deutscher Praktiken wie der dualen Berufsausbildung oder dem Aushandeln von Flächentarifverträgen nötig sind, wodurch Transfer mehr in einem deutschen Stil als in der Substanz sichtbar wird (Ferner/Varul 2000a; Ferner/Varul 2000b; Tüselmann et al. 2003; Meardi et al. 2009, 492). In Gastländern, die weniger stark reguliert sind als Deutschland und Firmen bezüglich ihrer HRM und IB Praktiken mehr Freiraum lassen, haben viele deutsche MNU zudem Gebrauch von dieser Freiheit gemacht, indem sie ihre Praktiken in Auslandsniederlassungen bewusst differenziert haben. Dies betrifft mehr die betriebliche Mitbestimmung als die duale Berufsausbildung. In diversen Gastländern wurde versucht, entsprechende Ausbildungspraktiken zu transferieren oder unter Nutzung lokal verfügbarer Infrastrukturen funktionale Äquivalente zu schaffen (Bluhm 2001, 161; Meardi et al. 2009, 507). Duale Ausbildungspraktiken wurden teilweise mehr als eine erprobte Praktik zur Rekrutierung und frühzeitigen Bindung junger Arbeitskräfte an das Unternehmen denn als reine Ausbildungsmassnahme transferiert (Bluhm 2001; Ferner et al. 2001, 118; Dickmann 2003). Hierbei war der Transfer aufgrund fehlender lokaler institutioneller Unterstützung und flexiblerer externer Arbeitsmärkte nach Grossbritannien (Ferner et al. 2001, 116; Dickmann 2003, 280) schwieriger als etwa nach Spanien (Ferner et al. 2001, 118). Ein weiteres Merkmal deutschen HRMs ist die langfristige Bindung und interne Entwicklung insbesondere technischer Fähigkeiten der Mitarbeiter (Dickmann 2003, 267). Dieses Merkmal spiegelt sich unter anderem in den oben geschilderten Praktiken der dualen Berufsausbildung sowie internen Karrieren, die auf wachsender technischer Expertise aufbauen, wider (Faulkner et al. 2002, 119-120). Eine kooperative Philosophie zeigte sich in deutschen $\mathrm{MNU}$ in ihrer grundsätzlich ausgeprägteren Verhandlungsbereitschaft wie etwa bei der Einführung flexibler Arbeitszeitkonten, was den von Wever $(1995,611)$ als „'negotiated' approach to employment relations“ bezeichneten Ansatz widerspiegelt. Ein solcher Ansatz wird auch darin deutlich, dass im Unterschied etwa zu ihren US-amerikanischen Pendants in deutschen MNU generell keine Anzeichen für eine zentral vorgegebene anti-gewerkschaftliche Politik gefunden wurden, und dass anderweitige Praktiken wie etwa in Grossbritannien vielmehr dem dezentralen Ansatz im Bereich HRM und IB zugeschrieben wurden (Ferner et al. 2001, 116).

Eine weitere wichtige Erkenntnis aus vorangegangenen Studien betrifft die Feststellung, dass viele der sich vergleichsweise spät internationalisierenden deutschen MNU von den HRM Praktiken der diesbezüglich weiter fortgeschrittenen angelsächsischen Firmen gelernt haben. Dieser Prozess wurde als „Anglo-Saxonization“ (Ferner/Quintanilla 1998) beschrieben und macht sich in deutschen MNU unter anderem in der Einführung angelsächsischer Tools zur direkten Mitarbeiterkommunikation wie standardisierten Umfragen 
oder leistungsabhängiger Entlohnung bemerkbar. Eine jüngere Studie fand nach wie vor klare Anzeichen für eine Fortführung dieses Trends im Bereich des HRM, wo insbesondere US-amerikanische MNU als Vorbilder fungieren (Pudelko/Harzing 2007). Hierbei findet ein solches Lernen allerdings „in the German manner“ (Ferner/Quintanilla 1998, 724) statt, da angelsächsische Konzepte wie etwa Shareholder Value in einen deutschen Kontext übersetzt werden, wodurch sich deren konkreter Inhalt und Bedeutung stark verändern können.

Wie wir oben gesehen haben, ist der erfolgreiche Transfer von einigen typisch deutschen Praktiken wie der dualen Berufsausbildung, langfristiger Personalentwicklung und interner Karrieren sowie kooperativer Praktiken im Bereich der industriellen Beziehungen abhängig von den jeweiligen Gegebenheiten im Gastland. Das Beispiel der dualen Berufsausbildung belegt hierbei die Wichtigkeit breit angelegter institutioneller Unterstützung und entsprechender lokaler Arbeitsmärkte für das Funktionieren solcher Praktiken. Ebenso ist der Transfer eines kooperativen Ethos (Ferner et al. 2001, 109) sicherlich leichter in einen Gastlandkontext hinein, der von kooperativen industriellen Beziehungen und sozialpartnerschaftlichen Traditionen geprägt ist.

Hinsichtlich ihrer zentralen Bedeutung für HRM Praktiken in Deutschland wurden unterdessen wiederholt die folgenden drei Schlüsselinstitutionen des deutschen Arbeitsmarktes hervorgehoben: duale Berufsausbildung, Kollektivverhandlungen und Mitbestimmung (Müller 1999, 126; Wächter/Müller-Camen 2002, 80; Giardini et al. 2005, 63). Für die vorliegende Untersuchung erscheint es daher naheliegend, Unterschiede sowie Gemeinsamkeiten zwischen Deutschland und der Schweiz anhand einer vergleichenden Betrachtung dieser drei Schlüsselinstitutionen sowie deren möglichen Auswirkungen auf HRM und IB Praktiken zu analysieren.

\section{Deutsche und Schweizer Institutionen des Arbeitsmarktes im Vergleich}

In den folgenden Abschnitten werden die Schlüsselinstitutionen des deutschen und schweizerischen Arbeitsmarktes vergleichend analysiert und auf ihre jeweiligen Einflüsse auf HRM und IB hin untersucht. Die in den folgenden Abschnitten genauer erörterten Gemeinsamkeiten und Unterschiede in den Bereichen duale Berufsbildung, Kollektivverträge, betriebliche Mitbestimmung und Arbeitsrecht bilden anschliessend den Rahmen für die folgende Fallstudienanalyse.

\subsection{Duale Berufsausbildung}

Deutschland und die Schweiz weisen grosse Ähnlichkeiten bezüglich der Berufsausbildung auf. Die Systeme beider Staaten entsprechen dem Typus der auf den Arbeitsplatz ausgerichteten und staatlich regulierten Systeme (Winterton 2007, 284). Dabei stellt sowohl in Deutschland (Berufsbildungsgesetz (BBiG) 2007) als auch in der Schweiz (Bundesgesetz über die Berufsbildung 2002) jeweils ein Gesetz die Regelungsgrundlage dar, welche Ausbildungsinhalte einheitlich regelt und somit für eine landesweite Standardisierung von Fertigkeiten und Fähigkeiten der Lehrlinge sorgt. In beiden Fällen wird das duale Ausbildungssystem durch ein tripartites System gesteuert, in dem die Definition von Ausbildungsinhalten sowie die Aufteilung der Lasten gemeinsam von Arbeitgeberverbänden, Gewerkschaften und dem Staat wahrgenommen werden (Hanhart/Bossio 1998, 485-486; Müller 1999, 126-127; Gonon 1999; Gonon 2005; Meunier 2007, 11). Der schulische 
Teil der Ausbildung wird in beiden Ländern von der öffentlichen Hand getragen, während die Unternehmen den praktischen Teil der Ausbildung durchführen und die entsprechenden Infrastrukturen und Lehreinheiten am Arbeitsplatz finanzieren (Hanhart/Bossio 1998, 492; Scharnhorst 2013, 18). In beiden Staaten ist durch dieses öffentliche Berufsbildungssystem das Mass an Autonomie jedes einzelnen Unternehmens in Fragen der Qualifikation stark eingeschränkt (Giardini et al. 2005). Wenngleich die Unternehmen über ihre Verbände Einfluss auf die kollektive Gestaltung von Lehrgängen und deren Ausbildungsinhalte nehmen, so ist der Spielraum des einzelnen Betriebes für nachträgliche Anpassungen an seine individuellen Bedürfnisse durch die verbindliche Definition von Ausbildungsform und -inhalten auf einen klar festgelegten Rahmen beschränkt. Auch hinsichtlich ihrer Bedeutung für die auf den Arbeitsmärkten breit verfügbaren Qualifikationen hat die duale Berufsausbildung mit einem Anteil von aktuell ca. 50\% in Deutschland (Destatis-Statistisches Bundesamt 2012, 23) sowie 70\% in der Schweiz (Eidgenössisches Departement für Wirtschaft 2013, 14) in beiden Staaten grundlegende Bedeutung. Sousa-Poza $(2004,45)$ unterstreicht in diesem Zusammenhang die grosse Bedeutung der dualen Ausbildung und entsprechend hoher Qualifikationsniveaus für die Wertschätzung und das hohe Mass an Vertrauen, das Unternehmen ihren Mitarbeitern entgegenbringen. Ebenso ging aus einer Studie von Hanhart und Bossio (1998, 496-497) hervor, dass die spezifisch auf die Bedürfnisse der Unternehmen ausgerichteten Kompetenzen der Lernenden einen Hauptgrund für das Engagement in der Lehrlingsausbildung darstellen. Hierdurch wiederum sind die Unternehmen stark an einem Schutz ihrer Investitionen in spezifisches Humankapital interessiert, da durch die Vermeidung von Rekrutierungs- und Einarbeitungskosten sowie die betriebsspezifische Ausbildung Fachkräfte mit Qualifikationen hervorgebracht werden, die zu entsprechenden Löhnen auf dem externen Arbeitsmarkt nicht zu finden wären (Müblemann/Wolter 2007, 44-45).

Ein bedeutender Unterschied zwischen Deutschland und der Schweiz besteht jedoch darin, dass Lehrlinge im deutschen dualen System während ihrer Ausbildungszeit für die Ausbildungsbetriebe Nettokosten verursachen, denen in der Schweiz ein Nettonutzen gegenübersteht (Wolter/Schweri 2003; Hoeckel 2008, 11-12; OECD 2010, 202). Eine Erklärung hierfür kann in der unterschiedlichen Zeitaufteilung in der Lehre gesehen werden, wobei die produktiven Zeiten der Lehrlinge in der Schweiz bei 53\% gegenüber lediglich $31 \%$ in Deutschland liegen (Wolter 2005, 21). Wolter $(2005,21)$ erklärt diesen signifikanten Unterschied in der Zeitaufteilung wiederum damit, dass Schweizer Betriebe sich bereits länger mit den Herausforderungen eines flexiblen externen Arbeitsmarktes konfrontiert sehen. Da insbesondere kleine und mittelständische Unternehmen nicht automatisch damit rechnen können, dass Lehrlinge ihnen nach der Ausbildung die Treue halten und die Betriebe anschliessend durch längeren Arbeitseinsatz die Ausbildungskosten amortisieren können, mussten sie ihre Ausbildungsmodelle den Gegebenheiten des lokalen Arbeitsmarktes anpassen.

\subsection{Kollektivverträge und industrielle Beziehungen}

Der gewerkschaftliche Organisationsgrad betrug im Jahr 2009 17.8\% in der Schweiz bzw. $18,8 \%$ in Deutschland und lag damit jeweils nahe dem Durchschnittswert der OECD-Länder (OECD 2012b). Sowohl in Deutschland als auch in der Schweiz sind Kollektivverträge ein zentrales Instrument zur Koordination der Arbeitsmärkte. Dennoch bestehen diesbezüglich einige wichtige Unterschiede. 
In Deutschland werden Tarifverträge traditionell auf regionaler und Branchenebene ausgehandelt und schliessen Lohnverhandlungen mit ein (Flanagan 1999; Visser 2006), wobei seit den 1990er Jahren ein anhaltender Trend hin zur Verlagerung auf die Firmenebene zu verzeichnen ist (Tijdens/van Klaveren 2007, 4; Guertzgen 2009, 326). Hinsichtlich der Auswirkungen des deutschen Systems der Tarifverhandlungen auf HRM Praktiken sprechen Giardini et al. (2005, 71) von einer Zweiteilung der Mitarbeiterschaft in tarifliche und aussertarifliche Angestellte, wobei die Managementprärogative für tarifliche Angestellte in zentralen Bereichen durch erhebliche Mitbestimmungsrechte des Betriebsrats stark eingeschränkt ist.

In der Schweiz werden Gesamtarbeitsverträge (GAV) zwar kleinräumiger, aber ebenfalls auf regionaler und Branchenebene ausgehandelt. Lohnverhandlungen finden in den wichtigsten Branchen jedoch regelmässig dezentral auf Unternehmens- oder Betriebsebene statt, was von einer informellen Koordination auf Arbeitgeberseite begleitet wird (Soskice 1990, 41; Flanagan 1999, 1152; Kenworthy 2003, 41). Der Trend seit Mitte der 1990er Jahre geht im Gegensatz zu Deutschland allerdings in Richtung höherer GAV-Deckungsraten. Diese Entwicklung steht in direktem Zusammenhang mit dem EU-Personenfreizügigkeitsabkommen und den flankierenden Massnahmen zur Verhinderung von Lohndumping. Hierzu wurden als Novum in den Schweizer IB in bestimmten Branchen wie dem Baugewerbe und der Gastronomie GAV mit Mindestlöhnen ausgehandelt und für allgemein verbindlich erklärt (Fischer 2003; Fumagalli-Senn 2009, 9-10). Der tarifvertragliche bzw. GAV Deckungsgrad liegt in der Schweiz derzeit bei etwa 45\% und in Deutschland bei ca. $65 \%$ (Venn 2009, 16, 18; Hayter et al. 2011, 227). Auf dem von Kenworthy $(2003,41)$ erstellten Index zur Lohnkoordination werden beide Länder in die selbe Kategorie relativ stark zentralisierter Systeme mit einem Wert von vier auf einer fünfstufigen Skala eingeordnet.

Ein zentrales Merkmal der wichtigsten Schweizer GAV ist zudem die Vereinbarung des absoluten Arbeitsfriedens (Schmid 2001, 451; Aubert 2005, 150), wodurch Streiks auch in vom GAV nicht erfassten Belangen während der Laufzeit ausgeschlossen sind (Aubert 2005, 150; Ackermann 2008; Broussolle 2009). Closed Shops und politische Streiks sind in beiden Ländern unzulässig (Aubert 2005, 149, 151). Hinsichtlich der Konfliktintensität der IB bekleidet die Schweiz dem entsprechend einen internationalen Spitzenplatz mit dem geringsten Mass an verlorenen Arbeitstagen durch Streiks pro 1000 Mitarbeitern und Jahr, wobei auch Deutschland weit unter dem OECD-Durchschnitt liegt (Lesch 2003, 31; Scheuer 2006).

Diese Zahlen spiegeln in beiden Ländern konsensorientierte sozialpartnerschaftliche Traditionen wider (Warner/Campbell 1993, 89-90; Friel 2005, 195). Einige Autoren (Schmid 2001, 452; Broussolle 2009, 292) weisen darauf hin, dass die schweizerische Form der Kooperation zwischen Sozialpartnern auf einem hohen Mass an Vertrauen und der Annahme von Gegenseitigkeit beruht, und dass bei seiner Durchsetzung soziale Kontrolle eine wesentliche Rolle spielt. Der Arbeitsfriede ist somit weniger als rechtliches denn als politisch-kulturelles Phänomen zu verstehen (Aubert 2005, 151).

\subsection{Betriebliche Mitbestimmung}

Hinsichtlich der Mitbestimmung bestehen grundlegende Unterschiede zwischen Deutschland und der Schweiz. 
In Deutschland räumt die gesetzliche Grundlage des Betriebsverfassungsgesetzes von 1972 dem Betriebsrat weitreichende Mitbestimmungsrechte in zentralen Bereichen des HRM wie Arbeitszeit, Arbeitsplanung, Boni und Leistungsziele für Mitarbeiter, Zahlung von Löhnen, berufliche Weiterbildung am Arbeitsplatz sowie Mitarbeiterdaten und -kontrolle ein (Schmitt 2003, 355; Williams/Geppert 2006, 57).

Obgleich man in der Schweiz das Mitwirkungsgesetz von 1993 kennt, so bleiben hier echte Mitbestimmungsrechte doch klar auf Fragen in Zusammenhang mit Pensionsfonds beschränkt, wobei ansonsten lediglich Informations- und Konsultationsrechte vorgesehen sind (Bonvin 2007, 37).

Das Schweizer Mitwirkungsgesetz ist hinsichtlich seiner formal rechtlichen Auswirkungen auf HRM Praktiken daher keineswegs mit der deutschen Mitbestimmung gleichzusetzen und schränkt die Entscheidungsmacht des Managements in Schweizer Firmen in Fragen des HRM kaum ein.

\subsection{Arbeitsrecht}

Wir möchten dieser vergleichenden Analyse der drei Schlüsselinstitutionen des Arbeitsmarktes mit einer Betrachtung des Arbeitsrechts noch eine weitere Kategorie hinzufügen, die wir in diesem Zusammenhang für wesentlich erachten.

In Deutschland spricht man im Bereich des HRM von einer starken Tendenz zur Verrechtlichung (Wächter/Stengelhofen 1992, 25) bzw. allgemein einem höheren Mass an formaler Regulierung (Avery et al. 1999). Dies schlägt sich nicht nur in den oben erwähnten gesetzlich festgeschriebenen Mitbestimmungsrechten des Betriebsrats, sondern auch in einem hohen Niveau an rechtlichem Kündigungsschutz nieder.

Ein zentraler Unterschied besteht hier zu dem stark ausgebauten Kündigungsschutz für reguläre Beschäftigungsverhältnisse und der Pflicht zur Ausarbeitung von Sozialplänen mit entsprechend hohen Kosten bei Massenentlassungen in Deutschland (Tempel 2001, 207; Royle 2004; Williams/Geppert 2006, 48; Venn 2009, 8; Dörrenbächer/Gammelgaard 2011, 34). Diesem steht in der Schweiz ein sehr liberales Arbeitsrecht mit geringem gesetzlichen Kündigungsschutz und ohne Verpflichtung zur Ausarbeitung von Sozialplänen bei Massenentlassungen gegenüber (Aubert 2005; Bonvin 2007; Venn 2009, 8). Während Kündigungen in Deutschland dementsprechend sehr langwierig und kostspielig sind (Giardini et al. 2005, 73), sind Entlassungen in der Schweiz rechtlich gesehen durch das Prinzip der Kündigungsfreiheit (Mahon 2000, 13) schnell und unkompliziert möglich. Der Schweizer Arbeitsmarkt gilt daher als äusserst flexibel (Bender et al. 2007, 172) oder gar als „beschäftigungspolitisches Erfolgsmodell“ (Nikolai 2005, 194, 197).

Betrachten wir diese Unterschiede vor dem Hintergrund der von Streeck (1987) diskutierten Unterscheidung zwischen Status und Vertrag als Grundkategorien in den industriellen Beziehungen, so wird ein grosser Unterschied zwischen Deutschland und der Schweiz deutlich. Während in Deutschland bei der Regulierung des Arbeitsmarktes wesentlich stärker auf formale rechtliche Vorschriften und damit auf das Statusprinzip gesetzt wird, so finden wir in der Schweiz eine stärker subsidiäre und auf flexiblere vertragliche Verhältnisse ausgerichtete Auslegung vor. Dies betrifft den Bereich der Lohnverhandlungen ebenso wie die betriebliche Mitbestimmung und Arbeitsrecht. 


\begin{tabular}{|c|c|c|c|c|}
\hline Land & $\begin{array}{l}\text { Duale Berufsaus- } \\
\text { bildung }\end{array}$ & $\begin{array}{l}\text { Kollektivverträge und industrielle } \\
\text { Beziehungen }\end{array}$ & Mitbestimmung & Arbeitsrecht \\
\hline D & $\begin{array}{l}\text { Auf den Arbeits- } \\
\text { platz ausgerichte- } \\
\text { tes, staatlich regu- } \\
\text { liertes duales Aus- } \\
\text { bildungssystem: } \\
\text { „workplace- } \\
\text { focused, state- } \\
\text { regulated system of } \\
\text { dual VET“ } \\
\text { (Winterton } 2007 \text {, } \\
\text { 284) }\end{array}$ & $\begin{array}{l}\text { Tarifverträge auf regionaler und } \\
\text { Branchenebene, kollektive Lohn- } \\
\text { verhandlungen (Flanagan 1999; } \\
\text { Visser 2006), seit 1990er-Jahren } \\
\text { Trend zur Firmenebene } \\
\text { (Tijdens/van Klaveren 2007, 4; } \\
\text { Guertzgen 2009, 326), allgemein } \\
\text { kooperative, wenig konfliktbelade- } \\
\text { ne Beziehungen (Lesch 2003, 31) }\end{array}$ & $\begin{array}{l}\text { Mitbestimmungsrechte } \\
\text { der Betriebsräte in zen- } \\
\text { tralen Bereichen des } \\
\text { HRM wie z.B. Arbeits- } \\
\text { zeit, Vergütung, beruf- } \\
\text { liche Weiterbildung } \\
\text { (Betriebsverfassungs- } \\
\text { gesetz 1972; Schmitt } \\
\text { 2003, 355; Williams/ } \\
\text { Geppert 2006, 57) }\end{array}$ & $\begin{array}{l}\text { Verrechtlichung } \\
\text { (Wächter/Stengelho- } \\
\text { fen 1992, 25; Avery } \\
\text { et al. 1999) Relativ } \\
\text { hohe Regelungs- } \\
\text { dichte und hohes } \\
\text { Niveau an gesetzli- } \\
\text { chem Arbeitsschutz } \\
\text { über OECD-Durch- } \\
\text { schnitt (Venn 2009) }\end{array}$ \\
\hline $\mathrm{CH}$ & $\begin{array}{l}\text { „workplace- } \\
\text { focused, state- } \\
\text { regulated system of } \\
\text { dual VET“ } \\
\text { (Winterton } 2007 \text {, } \\
284 \text { ) }\end{array}$ & $\begin{array}{l}\text { Gesamtarbeitsverträge (GAV) auf } \\
\text { regionaler Ebene, Lohnverhand- } \\
\text { lungen dezentral auf betrieblicher } \\
\text { Ebene mit Ausnahme von GAV- } \\
\text { Mindestlohnbestimmungen; abso- } \\
\text { luter Arbeitsfriede in wichtigsten } \\
\text { GAV festgeschrieben (Aubert } \\
2005,150 ; \text { Ackermann 2008; } \\
\text { Broussolle 2009), allgemein ko- } \\
\text { operative, wenig konfliktbeladene } \\
\text { Beziehungen (Lesch 2003, 31) }\end{array}$ & $\begin{array}{l}\text { Hauptsächlich Infor- } \\
\text { mations- und Konsul- } \\
\text { tationsrechte; Mitbe- } \\
\text { stimmung der Arbeit- } \\
\text { nehmer beschränkt } \\
\text { sich ausschliesslich auf } \\
\text { Fragen in Zusammen- } \\
\text { hang mit Pensions- } \\
\text { fonds (Mitwirkungsge- } \\
\text { setz 1993; Bonvin } \\
2007,37)\end{array}$ & $\begin{array}{l}\text { Subsidiaritätsprin- } \\
\text { zip und Arbeitsfrie- } \\
\text { de als Kernelemente } \\
\text { der Arbeitsmarktre- } \\
\text { gulierung: Weitge- } \\
\text { hende Vertragsfrei- } \\
\text { heit und Regelung } \\
\text { auf sozialpartner- } \\
\text { schaftlicher oder be- } \\
\text { trieblicher Ebene } \\
\text { (Bonvin 2007, 38) }\end{array}$ \\
\hline
\end{tabular}

Tabelle 2: Schlüsselinstitutionen des Arbeitsmarktes im deutsch-schweizerischen Vergleich (Quelle: Eigene Darstellung)

In der vergleichenden Analyse der wichtigsten Institutionen des Arbeitsmarktes und deren jeweiligen Merkmalen wird deutlich, dass beide Länder sehr ähnliche Systeme der dualen Berufsausbildung vorweisen (s. Tabelle 2). In den anderen Bereichen sind hingegen grössere Unterschiede festzustellen, was dazu geführt hat, dass die Schweiz jüngst von einigen Autoren als hybride (Teuber/Backes-Gellner 2013) oder liberale Marktwirtschaft (Schneider/Paunescu 2012) klassifiziert wurde.

\section{Methodik}

Die vorliegende Studie ist Teil eines breiter angelegten, mehrjährigen Forschungsprojektes über den Transfer von HR Praktiken in MNUs, in dessen Rahmen bereits über 30 schweizerische Auslandsniederlassungen von MNUs deutscher, amerikanischer und französischer Herkunft untersucht wurden.

Die Studie basiert auf der qualitativen, multiplen Fallstudien-Methode (Yin 2009), was im Bereich der Forschung zum internationalen Transfer von HRM Praktiken in MNUs den derzeit am weitesten verbreiteten Ansatz darstellt (Ferner 2001; Tempel 2001; Wächter et al. 2003; Dickmann 2003; Ferner et al. 2004; Wächter/Peters 2004b; Almond et al. 2005; Edwards et al. 2005; Tempel et al. 2006). Qualitative Daten eröffnen einen Einblick in komplexe soziale Prozesse (Eisenhardt/Graebner 2007, 26) und sind daher besonders geeignet für die Analyse des internationalen Transfers von HRM Praktiken in MNUs mit seiner Vielzahl an Einflussfaktoren. Die Stärke dieser Forschungsstrategie liegt gegenüber quantitativen Ansätzen insbesondere in ihrer internen- sowie Konstruktvalidität, die wiederum im Sinne einer Hierarchie die Basis für externe Validität und Reliabilität bilden (Gibbert et al. 2008, 1468). Ähnlich wie in den vorangegangenen Studien, die in Ab- 
schnitt 2. vorgestellt wurden, haben wir die Zusammenhänge zwischen institutionellem Umfeld und organisationalen Praktiken untersucht. Die interne Validität der Studie wird hierbei durch ein theoriegeleitetes, strukturiertes Vorgehen sichergestellt (Wächter 2004, 3).

Für die vorliegende Studie wurden im Zeitraum zwischen Dezember 2008 und Juni 2011 insgesamt 19 Interviews mit HR Managern sowie deutschen Expatriierten in schweizerischen Auslandsniederlassungen von neun deutschen MNUs verschiedener Branchen und Grössenklassen geführt (siehe Tabelle 3). Diese Heterogenität ist im Sinne der Beobachtbarkeit qualitativ unterschiedlicher Anforderungen und Probleme sowie unterschiedlicher Sichtweisen aus theoretisch-methodischer Sicht erwünscht (King 2004a, 16-17). Da in einem multiplen Fallstudiendesign die analytische und nicht statistische Verallgemeinerbarkeit erreicht werden soll, war für die Auswahl der Fälle das Kriterium der Diversität und nicht dasjenige der Repräsentativität entscheidend (Hartley 2004, 331).

Zwecks Erreichens analytischer Verallgemeinerbarkeit durch Vergleiche zwischen Fällen sowie einer ausreichenden Zahl beobachtbarer Phänomene und Variablen werden vier bis zehn Fälle als gute Basis genannt (Eisenhardt 1989). In diesem Zusammenhang wurde auch darauf hingewiesen, dass ein Vergleich der Ergebnisse aus der Fallstudie mit der Literatur einen erheblichen Beitrag zur analytischen Verallgemeinerbarkeit und Reliabilität leistet (Eisenhardt 1989; Hartley 2004), was durch unsere Zusammenfassung bisheriger Forschungsergebnisse und Diskussion der neuen Ergebnisse vor diesem Hintergrund gewährleistet wird.

Die halb strukturierten Interviews mit HR Managern hatten eine Dauer von 90-120 Minuten, und basierten auf dem an den Schweizer Kontext angepassten Interviewleitfaden aus der Studie von Wächter et al. (2003; 2004a). Die Interviews mit Expatriierten hatten eine Dauer von 60 bis 90 Minuten. Hierbei wurden alle Gespräche in der Muttersprache des Interviewpartners - deutsch oder französisch - geführt, um das Gespräch für die Interviewpartner so leicht und angenehm wie möglich zu gestalten (Marschan-Piekkari/Reis 2004). Deutschschweizern, die sich oftmals lieber in ihrer Mundart ausdrückten, wurde dies explizit freigestellt.

Bei allen Interviews handelt es sich im Sinne von King (2004a, 12) um „realist interviews“, wobei die in den Interviews erhaltenen Informationen als Einsicht in das organisationale Leben ausserhalb der Interviewsituation behandelt werden. Gesprächstranskripte wurden durch die Interviewpartner kontrolliert und validiert, und anschliessend nach dem Verfahren der „template analysis“ (King 2004b, 256) anhand von MAXQDA analysiert, um ein grösstmögliches Mass an Reliabilität, Transparenz und Nachvollziehbarkeit zu gewährleisten (Sinkovics et al. 2008). Die template analysis der Interviews war hierbei auf die Identifikation von Zusammenhängen zwischen organisationalen HRM und IB Praktiken sowie institutionellem Umfeld fokussiert. Hierbei spielte zur Sicherung hoher Konstruktvalidität Triangulierung durch Vergleich mit Daten aus quantitativen Forschungen sowie der Dokumentenanalyse eine bedeutende Rolle. Dieser Forderung wurde mittels Hinzuziehung der Jahresberichte, weiterer veröffentlichter Dokumente, via Internetrecherche sowie der Durchführung von vier zusätzlichen externen Experteninterviews und Analyse durch zwei Forscher nachgekommen 


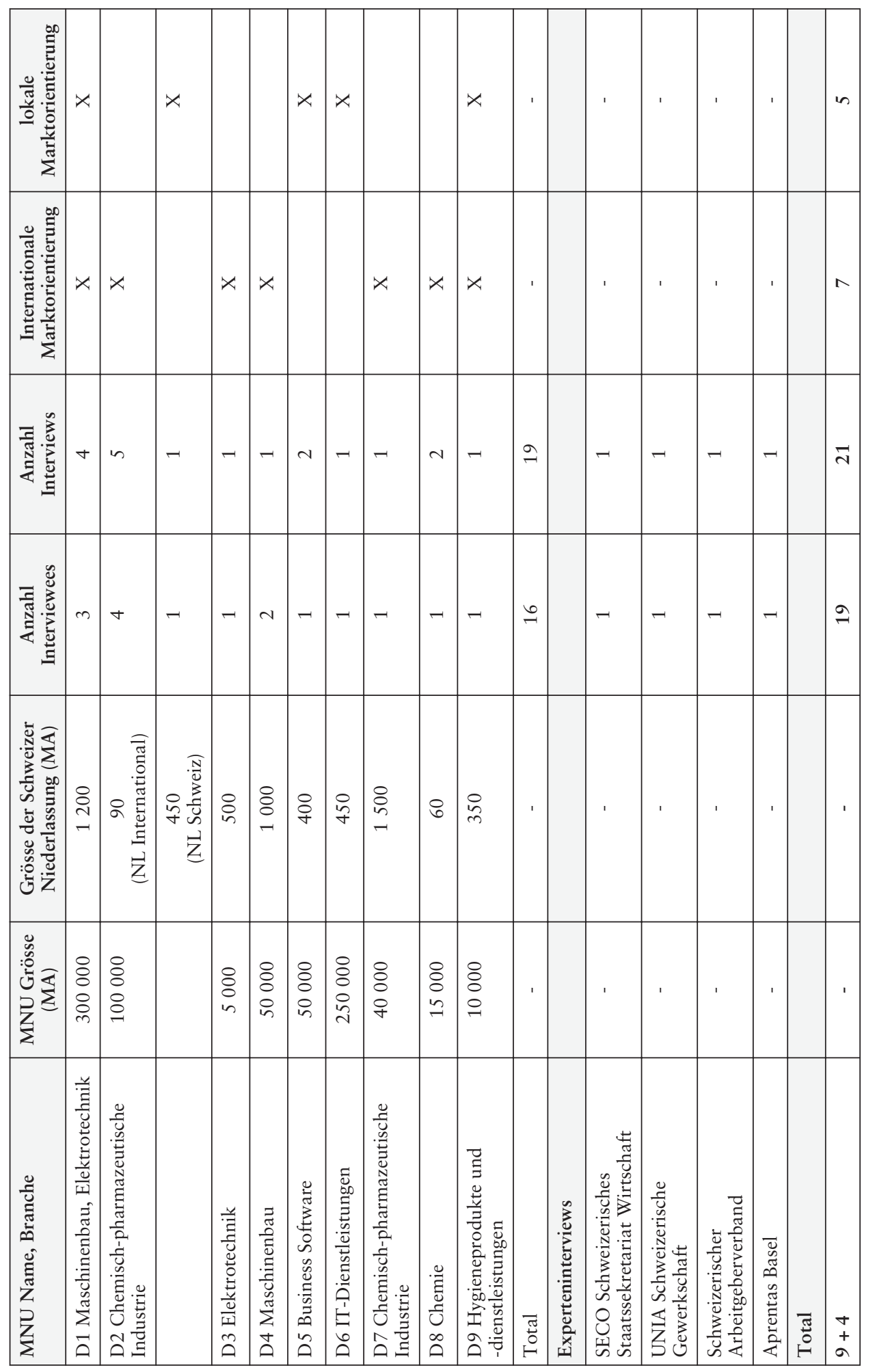

章

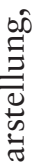

ت

.

$\stackrel{\ddot{0}}{\underline{0}}$

章

武

है

तี

$\Xi$

它

Z

寻

我

e

远

$\because \frac{1}{1}$

ป 혼

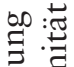

劳

艺

는

:ี

:

$\ddot{m} \dot{0}$

을

$\approx$ E 


\section{Resultate der empirischen Studie: Lokale HRM und IB Praktiken deutscher MNU in der Schweiz}

Die Resultate unserer empirischen Forschung werden im Folgenden untergliedert in die Bereiche duale Berufsausbildung, industrielle Beziehungen und Mitbestimmung.

\subsection{Berufsausbildung}

In den dualen Ausbildungspraktiken deutscher MNU spiegeln sich konkret und deutlich die diesbezüglichen Ähnlichkeiten mit dem Schweizer System wider. Acht der neun von uns untersuchten deutschen MNU in der Schweiz bilden Lehrlinge aus, wobei auch in dem einzigen Unternehmen, das aktuell keine Lehrlinge ausbildet, laut unserem Interviewpartner alle nötigen Infrastrukturen vorhanden sind und man durchaus willens ist, wieder Lehrlinge auszubilden. Besonders interessant erscheint hierbei auch, dass D2 und D4 aktive Mitglieder in einem Schweizer Ausbildungsverbund sind. Solche lokalen Strukturen können als ein Beispiel für auf subnationaler Ebene zur Verfügung gestellte „supports“ für MNU interpretiert werden (Almond 2011, 532-533), die durch Bündelung der Kräfte die Realisierung von Skaleneffekten ermöglichen. Der Aspekt der hiermit verbundenen erheblichen Kosteneinsparungen für die teilnehmenden Unternehmen wurde in unserem Interview beim Basler Ausbildungsverbund Aprentas besonders deutlich: „Je mehr sich beteiligen, umso günstiger wird das. Und wir sind gestartet mit diesen drei Trägerfirmen (...) in der Zwischenzeit schwanken wir so zwischen ca. 65-70 Mitgliederfirmen. (...) Ein Lernender kostet bei uns ca. 40\% von dem was [Nichtmitgliedsfirma] für einen Lernenden ausgibt" (Aprentas: 20). Interessant ist hierbei auch die Feststellung unseres Interviewpartners, dass z.B. amerikanische Mitgliedsfirmen ursprünglich schweizerische oder deutsche Wurzeln hatten: „(...) es sind keine, ich sage mal (...) amerikanischen Firmen, die hier sich neu niederlassen und dann zu uns kommen. Es haben alle Schweizer Wurzeln, oder deutsche" (Aprentas: 146). Hierin spiegelt sich deutlich die Vertrautheit deutscher MNU mit der dualen Ausbildung wider, die im Sinne eines country-of-origin Effekts direkt bereit sind, sich an einem solchen Verbund zu beteiligen, wohingegen diese Ausbildungspraxis etwa bei amerikanischen Firmen nicht üblich ist.

Betrachten wir nun noch etwas genauer die Gründe, die der Ausbildungsbereitschaft deutscher MNU in der Schweiz zugrunde liegen, so erhalten wir weitere interessante Einblicke. Während der Einsatz von Lehrlingen in Niederlassungen mit industrieller Produktion wie bei D1, D2 Schweiz, D3, D4 oder D9 naheliegend erscheint, so ist dies im Fall von D2 International oder D5 keineswegs der Fall. In der Tat weisen die Aussagen unseres D5Interviewpartners klar auf das Vorhandensein von nicht-rational ökonomischen Gründen für die lokale Lehrlingsausbildung hin, was sich mit Hinweisen auf positive Reputationseffekte aus vorangegangenen Studien deckt (Hanhart/Bossio 1998, 496-497; Wolter 2005, 21): „Wir haben Lernende. Wir halten das letzten Endes auch sehr, sehr hoch. Man muss hart formulieren. Wir brauchen es nicht, um zu überleben. Also wir bilden nicht aus, in dem Sinne, um einen speziellen eigenen Bedarf zu decken, sondern das hat viel auch einfach mit sozialer Verantwortung zu tun" (D5_I1no1: 239). Die Verbindung von Lehrlingsausbildung und sozialer Verantwortung in der Schweiz wurde auch in unserem Interview mit einem Vertreter des Schweizer Arbeitgeberverbands deutlich: „Es gehört ein Stück weit auch zur (...) Corporate Social Responsibility, wo wir sagen, kommt völlig selbstverständlich mit rein (...) ein anständiges Schweizer Unternehmen bildet auch Lehr- 
linge aus" (Schweizer Arbeitgeberverband: 95). Eine ähnliche Begründung fanden wir auch bei D9. Obwohl es sich hierbei um einen produzierenden Betrieb handelt, stellte unser Interviewpartner klar, dass neben rational-ökonomischen Argumenten ganz klar auch soziale Legitimität eine Rolle bei der Entscheidung spielte, im Rahmen einer Werksverkleinerung im Gegenzug die Zahl der Ausbildungsplätze zu verdoppeln (D9_I1no1: 524, 529-533).

Vorangegangene Studien hatten gezeigt, dass deutsche MNU bereits unter wesentlich ungünstigeren institutionellen Rahmenbedingungen versucht hatten, duale Berufsausbildung zu transferieren oder funktionale Äquivalente zu schaffen. Erwartungsgemäss investieren die von uns untersuchten Unternehmen unter den günstigen Schweizer Voraussetzungen in die Berufsausbildung, was mit der institutionellen Einbettung dieser Praktiken sowohl im Herkunfts- als auch im Gastland der MNU in Einklang steht.

\subsection{Industrielle Beziehungen und Kollektivverträge}

In keinem der Fallstudien-MNU wurden Anzeichen für anhaltende Konflikte in den IB in der Schweiz vorgefunden. Die deutschen MNU unserer multiplen Fallstudie folgen in weiten Teilen den lokal üblichen Praktiken. Dennoch sind die einzelnen Praktiken je nach MNU und Standort verschieden.

So fanden wir in der Hälfte der von uns untersuchten Fälle eine Personalkommission, die als Sozialpartner z.B. in die Lohnverhandlungen eingebunden wurde. Dies entspricht, wie die Aussage unseres D9-Interviewpartners illustriert, der traditionellen Schweizer Praxis: „Also wir haben natürlich eine Arbeitnehmerkommission [AK] (...) wir haben natürlich normal Sozialpartnerschaft mit unserer Arbeitnehmerkommission (...) Lohnverhandlungen werden auch mit der AK natürlich besprochen, Inputs kommen von dort, müssen auch gemeinsam getroffen werden. In dem Rahmen halt, in dem das in der Schweiz üblich ist, sage ich jetzt mal" (D9_I1no1: 604).

Lediglich vier unserer neun Fallstudien-MNU haben einen GAV unterzeichnet, wobei fünf Beziehungen mit Gewerkschaften unterhalten. Wichtig erscheint hierbei jedoch, dass auch in Fällen, in denen keine Beziehungen zu Gewerkschaften unterhalten werden, dies nicht auf eine ideologische anti-gewerkschaftliche Einstellung zurückgeführt werden kann: „(...) rein von der Denke her kommt das nicht, haltet mir die Gewerkschaft raus, weil Deutschland könnte das gar nicht. Das ist im Gegensatz zu amerikanischen Firmen natürlich eine ganz andere Denke. In Deutschland ist man gewohnt, sehr stark verhandeln zu müssen" (D1_Ino1: 218).

Bei den Fällen von D1 und D2 wird hierbei die Bedeutung der jeweils in der Schweiz stattfindenden Aktivitäten deutlich. Während in den Produktionsbereichen Beziehungen mit Gewerkschaften gepflegt und GAV ausgehandelt werden, ist dies erwartungsgemäss in denjenigen Unternehmensbereichen und Niederlassungen wie D2 International, D5, D8 oder D7, in denen hauptsächlich Manager und Arbeitnehmer mit Universitätsabschlüssen tätig sind, nicht der Fall.

Ein weiterer interessanter Fall, der auf eine Kombination aus deutschem country-of-origin und Schweizer host country Effekt schliessen lässt, stellt D1 dar. Hier musste in den vergangenen Jahren eine Werksverkleinerung vorgenommen werden, und das Management akzeptierte grosszügige Sozialpläne: „Also D1 hat da unheimlich viele Gelder bereitgestellt in den letzten Jahren, um noch Leute in den Vorrubestand zu schicken. Auf eine sehr faire Art, also wirklich. Das ist einerseits D1 (...) Hat aber natürlich auch den Hinter- 
grund auch, den deutschen, dass Abbau in Deutschland auch sebr teuer ist, so dass D1 da dann die deutsche Brille hat. Und wenn ich mit einem für Schweizer Verhältnisse sehr hohen Betrag komme und sage: wenn ihr es sozialverträglich machen wollt, kostet es so viel, muss aber nicht sein nach Schweizer Gesetz, aber es wäre fair, dass da D1 dann relativ schnell zusagt, wo ein Amerikaner sagen würde: bist du völlig verrückt?" (D1_I1no1: 233).

Diese Praxis entspricht also wie auch die Lehrlingsausbildung einer bewussten Suche nach sozialer Legitimität, die in diesem Fall durch Verhandlung mit der lokalen Gewerkschaft als Sozialpartner erlangt wird. Insbesondere für MNU haben in diesem Zusammenhang Kostova et al. $(2008,999,1001)$ auf die Möglichkeit hingewiesen, dass diese ihre soziale Legitimität bewusst als Teil eines „symbolic image building“ mit lokalen Akteuren verhandeln können. Der Fall von D1 könnte daher als ein solches Beispiel gesehen werden, das sozialpartnerschaftliche Traditionen widerspiegelt, die sowohl in Deutschland als auch in der Schweiz vorzufinden sind.

Generell erscheint eine klare Zuordnung von country-of-origin und host country Effekten im Bereich der industriellen Beziehungen schwierig. Die Ergebnisse unserer Fallstudie lassen es mitunter naheliegend erscheinen, dass verschiedene Mitarbeiterkategorien bzw. Aktivitäten der Schweizer Niederlassung als nicht an den institutionellen Kontext gebundenen organisationalen Einflussfaktoren eine bedeutende Rolle spielen.

\section{$5 \cdot 3$ Mitbestimmung}

In unseren Fallstudien wurden in der Schweiz erwartungsgemäss keine formalen Mitbestimmungsrechte gefunden. Dennoch weisen die Aussagen unserer Interviewpartner eindeutig darauf hin, dass Konsens und Mitarbeiterpartizipation in der Praxis omnipräsent sind: „Die Schweizer sind damit aufgewachsen, dass sie immer mitreden können. Die dürfen Volksabstimmungen machen (...) sie wollen mitreden“ (D1_I3no1: 180). „Ein wesentlicher Unterschied ist schon die Sorgfältigkeit der Abstimmung, die man hier am Standort in der Schweiz jetzt unternehmen muss. Also ich empfinde es schon so, dass die Schweizer (...) beim Umgang miteinander sehr darauf achten, wer wird wann wie gefragt? Wer kann wie seine Meinung äussern zu welchem Thema, frühzeitig?" (D4_I1,I2no1: 520)

Offensichtlich herrscht in der Schweiz auch ohne gesetzlichen Zwang de facto seitens der Mitarbeiter die klare Erwartung, in Entscheidungsprozesse aktiv eingebunden zu werden, und die Bereitschaft seitens des Managements, diesen Erwartungen nachzukommen: „(...) good citizen: nur weil man ein liberales Arbeitsrecht hat legitimiert das einem nicht sich zu verhalten wie ein Schwein. Weil man trifft sich immer zweimal im Leben (...) das Image, das man sich auf dem Arbeitsmarkt erarbeitet auch, das ist nicht einfach unwichtig (D6_I1no1: 285).

Hierbei werden zwei Dinge deutlich: zum einen die Präsenz persönlicher sozialer Kontrolle, da man seine Mitarbeiter in der kleinen Schweiz wieder und wieder trifft, und zum anderen die Bedeutung des flexiblen Schweizer Arbeitsmarktes. Die Dynamik des Arbeitsmarktes erlaubt es nicht nur einseitig den Arbeitgebern, Mitarbeiter problemlos zu entlassen, sondern sie übt auch disziplinierenden Druck auf die Unternehmen dahingehend aus, dass Mitarbeiter, die mit ihrem Arbeitgeber unzufrieden sind, jederzeit die Firma verlassen können: „Wenn sie bei uns unglücklich sind und wieder zehn gehen oder so, dann habe ich ein Problem. Also ich muss schon sehr viel vorsichtiger sein. (...) Da gibts einen liquiden Arbeitsmarkt, der wirklich funktioniert und im Deutschen ist das halt schon ein biss- 
chen weniger der Fall“ (D6_I1no1: 271, 273); „Zwischen Genf und Lausanne gibt es 70 MNU (...) Manchmal findet das Gesetz des Marktes Anwendung “ (D7_I1no1: 252, eigene Übersetzung). Auch in diesem flexiblen, durch geringen Kündigungsschutz gekennzeichneten Arbeitsmarkt sind Arbeitgeber offensichtlich an stabilen Arbeitsbeziehungen interessiert und entsprechend sensibel: „Eine grundlose Entlassung ist in der Schweiz schwer zu verstehen“ (D2_I1no2, I5no1: 8, eigene Übersetzung); „Wir hatten uns das vorher gut überlegt. Wir entlassen nicht einfach so Knall auf Fall unser Personal" (D3_I1no1: 320, eigene Übersetzung).

Ferner konnten wir einen klaren deutschen country-of-origin Effekt feststellen, der in direktem Zusammenhang mit dem Einfluss deutscher Gesamtbetriebsräte steht. In Deutschland muss das Management viele HRM Praktiken gerade im Bereich der Leistungsbeurteilung mit dem Betriebsrat verhandeln. Dessen Einfluss jedoch ist für die Kategorie tariflicher Angestellter wesentlich grösser als für aussertarifliche Angestellte. Dies führt dazu, dass in vielen deutschen MNU unserer Studie gerade im Performance Management unterschiedliche Systeme und Praktiken in den jeweils niedrigeren oder höheren Hierarchiestufen im Einsatz sind. Diese Tatsache, die klar Merkmale der deutschen Arbeitsmarktinstitutionen widerspiegelt, ist auch in den Schweizer Auslandsniederlassungen deutscher MNU sichtbar: „Also was mir typisch scheint (...) es gibt viele Dinge, denen man ansieht, wie, dass sie mit dem Gedanken gestrickt sind, der deutsche Betriebsrat muss zustimmen. (...) Eben so irgendwelche Beurteilungen, Instrumente, so die so oft daberkommen, dass man eben sieht: so, für die leitenden Angestellten, für die Manager ist es so und für die Übrigen ist es so, wo man genau sieht, das eine haben sie nicht mit dem Betriebsrat abstimmen müssen, das [andere] müssen sie aber" (D6_I1no1: 422, 424).

Ähnliches wurde auch in D1 berichtet: „Dann industrielle Beziehungen (...) ich sage mal, da drückt D1 schon durch, also man spürt, dass dann beispielsweise wenn die weltweites Kompetenzmanagement einführen wollen, fragen die zuerst mal in jedem Land nach und sagen: Was müssen wir noch mit dem Betriebsrat in ibrem Land tun, damit wir das umsetzen dürfen? Was einem Schweizer meist ein mildes Lächeln entlockt, und da sagen wir: nichts" (D1_I1no1: 200).

Die strengen gesetzlichen Vorgaben und Verhandlungszwänge in Deutschland haben also offensichtlich einen spürbaren Einfluss, der über die Sozialisierung deutscher Manager auch auf deren Normen, Denken und Handeln im Ausland einwirkt. Interessant ist in diesem Zusammenhang, dass sich diverse Schweizer Manager hinsichtlich eines als zu konsensuell empfundenen Stils deutscher Manager bei der Leistungsbeurteilung und Entscheidungsfindung kritisch äusserten: „(...) in diesem Performance, (...) in diesem Talentprozess, (...) dass man da nur die Potentialträger melden (...) und identifizieren darf. Also die Low-Performer, die guckt man sich nicht an (D6_I1no1: 405), ebenso (D4_I1,I2no1: 509)(D7_I1no1: 404)(D2_I1no1: 253).

Während die Schweiz keine formellen Institutionen im Bereich der Mitbestimmung aufweist, scheinen diesbezügliche Praktiken in den Tochtergesellschaften deutscher MNU von anderen Elementen des lokalen institutionellen Umfeldes beeinflusst zu werden. Unsere Interviews lassen es naheliegend erscheinen, dass in Abwesenheit von arbeitsrechtlichen Zwängen insbesondere die politische Kultur in einem direktdemokratischen Land eine entscheidende Rolle spielt. Ein weiterer plausibler Grund für die grosse Sensibilität der Arbeitgeber gegenüber den Erwartungen der Arbeitnehmer kann in der disziplinierenden Wirkung des flexiblen Schweizer Arbeitsmarktes gesehen werden. 


\section{Diskussion}

Die vorliegende Untersuchung liefert ein differenziertes Bild von HRM und IB Praktiken in Schweizer Niederlassungen deutscher MNU. Deutsche sowie Schweizer Einflüsse wurden mittels einer Analyse der Regulierung der Arbeitsmärkte in Zusammenhang mit ihrem jeweiligen institutionellen Umfeld gebracht. Auf diese Weise konnten sowohl country-of-origin als auch home country Effekte auf einzelne HRM Praktiken aufgezeigt werden.

Eine grosse Gemeinsamkeit besteht im Bereich der dualen Berufsbildung. Obgleich in Deutschland keinerlei gesetzliche Verpflichtung zur Lehrlingsausbildung besteht, so wird doch seitens Gewerkschaften und Politik gesellschaftlicher Druck auf Unternehmen ausgeübt, sich hieran aktiv zu beteiligen (Giardini et al. 2005, 70). Auch in der Schweiz wiesen bereits vorangegangene Studien auf die Existenz sozialer Gründe für die Lehrlingsausbildung hin (Hanhart/Bossio 1998, 496-497; Wolter 2005, 21). Dies lässt es naheliegend erscheinen, dass Unternehmen ebenfalls unter sozialem Druck stehen, sich an der Lehrlingsausbildung zu beteiligen, was als Teil der Corporate Social Responsibility gesehen wird. Die Ergebnisse der vorliegenden Studie zur Ausbildungsbereitschaft deutscher MNU in der Schweiz belegen, dass die Wahrnehmung sozialer Verantwortung durch die Betriebe nach wie vor eine ungebrochen grosse Rolle bei ihrer Ausbildungsbereitschaft spielt. Dieser Befund ist auch interessant vor dem Hintergrund der theoretischen Argumentation des Neoinstitutionalismus, der Isomorphismus im Sinne der strukturellen Angleichung an lokale Organisationsformen und Praktiken als Mechanismus zur Erlangung von gesellschaftlicher Legitimität sieht (Meyer/Rowan 1977; DiMaggio/Powell 1983). Die Lehrlingsausbildung scheint in der Schweiz in diesem Sinne von deutschen MNU bewusst genutzt zu werden, um soziale Legitimität zu erlangen. Jedoch haben unsere Ergebnisse auch gezeigt, dass rational-ökonomische Gründe im Sinne der Verfügbarkeit spezifischer Qualifikationen für die Ausbildungsbetriebe nach wie vor eine wichtige Rolle bei der Entscheidung für die Lehrlingsausbildung spielen (Hanhart/Bossio 1998; Mühlemann/Wolter 2007). Diese Faktoren wirken zusammen mit der grossen Wertschätzung einer breiten beruflichen Qualifikation der Arbeitskräfte in einem Umfeld, in dem der Managementstil ähnlich wie auch in Deutschland stark fachwissenbetont ist (Bergmann 1990, 368; Warner/Campbell 1993, 97; Bergmann 1994, 89). Gut qualifizierten Mitarbeitern wird grosses Vertrauen entgegengebracht und ein hohes Mass an Autonomie und Mitsprache in flachen Hierarchien zugestanden.

Der Umstand, dass in unserer Fallstudie keine Anzeichen für anti-gewerkschaftliche Ideologien seitens der deutschen Muttergesellschaften gefunden wurden, steht in Einklang mit früheren Erkenntnissen aus der Studie von Ferner et al. (2001, 116). Dieses Ergebnis weist ferner darauf hin, dass das Konzept der Sozialpartnerschaft nach wie vor bei deutschen Unternehmen präsent zu sein scheint und zumindest nicht vollständig von individualisierten Praktiken angelsächsischen Ursprungs abgelöst wurde. Zudem stützen die Ergebnisse unserer Untersuchung im Bereich IB diejenigen vorangegangener Studien, in denen eine Dezentralisierung dieser Praktiken in deutschen MNU festgestellt wurde. Die Einbeziehung von Personalkommissionen als Sozialpartner bei betrieblichen Lohnverhandlungen in der Hälfte der von uns untersuchten Tochtergesellschaften entspricht lokalen Schweizer Gepflogenheiten im Sinne eines host country Effekts und ähnelt weniger typisch deutschen Praktiken.

Wie wir gesehen haben, bleiben deutsche MNU auch in der liberalen Schweiz in Teilen von den Schlüsselinstitutionen des deutschen Arbeitsmarktes im Sinne eines country-of- 
origin Effekts beeinflusst. Dies gilt insbesondere für Verhandlungszwänge, die grundsätzlich präsent sind, wenn globale Praktiken wie Leistungsbeurteilung und Vergütung als Teile von Performance Management Systemen zentral formuliert werden sollen. Solche globalen Praktiken müssen mit dem heimischen deutschen Betriebsrat ausgehandelt werden, der für tarifliche Angestellte erheblich grösseren Einfluss hat als für aussertarifliche. Daher ist in den globalen Systemen deutscher MNU auch in der Schweiz diese Unterscheidung in den HR-Systemen und Praktiken zwischen verschiedenen Hierarchiestufen präsent. Ferner spiegeln sich die im deutschen System omnipräsenten Verhandlungszwänge mit Betriebsräten und Gewerkschaften auch in den sozialpartnerschaftlichen Ansätzen in Schweizer Niederlassungen wider. Bluhm $(2001,156)$ führt hierzu aus, dass institutionelle Wahl immer beschränkter Rationalität unterworfen ist, da Akteure zwar ihre Politik ändern können, jedoch nicht dazu in der Lage sind, ihre eigenen Erwartungen oder diejenigen der anderen vollständig zu kontrollieren. Dies gilt ebenso für die sozialen Normen und Routinepraktiken, an die sie gewohnt sind. Diese Prägung von Akteuren durch ihr institutionelles Umfeld wurde in der Literatur als „socialised rationality“ (Almond 2011, 260) oder „embedded managerial ideological norms“ (Almond 2011, 265) bezeichnet, was ebenfalls in konkreten HRM Praktiken und deren Umsetzung in der Schweiz zum Ausdruck kommt. Besonders interessant erscheint in diesem Zusammenhang, dass ausgerechnet die von einem deutschen konsensuellen Stil geprägten Praktiken der Leistungsbeurteilung oder Entscheidungsfindung in Schweizer Niederlassungen kritisch beurteilt wurden, obgleich die Schweiz für ihren konsensuellen Stil bekannt ist (Tixier 1994, 16; Weibler/Wunderer 2007, 278). Dies zeigt, dass Managementpraktiken und Herangehensweisen, die als typisch deutsch empfunden werden, in der Schweiz keineswegs kritiklos übernommen werden. So stellte hinsichtlich der Leistungsbeurteilung einer unserer Interviewpartner fest: „Die Schweizer sind kritischer, die Deutschen lauter“ (D5_I1no2: 29). Unsere Ergebnisse deuten daher darauf hin, dass deutsche Praktiken in der Schweiz, anders als dies in Spanien oder Osteuropa der Fall sein mag, keinen symbolischen Vorbildcharakter besitzen und grundsätzlich auf ihre Sinnhaftigkeit und Funktionalität hin kritisch hinterfragt werden.

In Bezug auf die in der Schweiz praktizierte Mitbestimmung konnten wir einen host country Effekt identifizieren, der in Zusammenhang mit der direktdemokratisch geprägten politischen Kultur des Landes steht. Letztere kommt in den Erwartungen der Mitarbeiter zum Ausdruck, an ihrem Arbeitsplatz genau wie in politischen Entscheidungen mitsprechen zu dürfen (Chevrier 2002). Hinzu kommt ein disziplinierender Effekt, der ganz im Sinne einer people-networking society wirkt (Avery et al. 1999, 22). In der kleinen Schweizer Gesellschaft führt das Gesetz des Wiedersehens (Erten et al. 2004, 120-121; Freitag 2004, 113; Weibler/Wunderer 2007, 283) dazu, dass ähnlich wie im Fall des Arbeitsfriedens soziale Kontrolle (Broussolle 2009, 292) effektiv funktioniert, ohne dass eine solche Verhaltenskontrolle wie in Deutschland gesetzlich abgesichert wäre. Die Effektivität dieses sozialen Kontrollmechanismus wird nochmals signifikant erhöht durch die Flexibilität des lokalen Arbeitsmarktes, der bei einer sehr niedrigen Arbeitslosenquote die Machtbalance zwischen Arbeitnehmer und Arbeitgeber dahingehend verschiebt, dass er ersteren die „exit option“ (Meardi 2007, 519; Meardi et al. 2009) eröffnet. Trotz des geringen Kündigungsschutzes und des sehr flexiblen Arbeitsmarktes, was Arbeitgebern viel Spielraum für hireand-fire Strategien und Praktiken lässt, erklärt das komplexe Zusammenspiel der oben dargestellten Einflüsse die in der Praxis grosse Stabilität der Schweizer Beschäftigungsver- 
hältnisse (Sousa-Poza 2004, 34, 44-45) und Sensibilität der Arbeitgeber beim Thema Entlassungen.

\section{Schlussfolgerungen}

Die Ergebnisse unserer Studie zeigen, dass wesentliche Ansätze und Verhaltensweisen deutscher MNU, wie sie in anderen Gastländern gefunden wurden, auch in der Schweiz präsent sind. Hinsichtlich ihres Transfers von Praktiken der dualen Berufsbildung, eines konsensuellen Stils sowie eines dezentralen Managements im Bereich der IB entsprachen die Resultate unsere Fallstudie weitgehend den Beobachtungen vorangegangener Studien.

Gleichzeitig wurde deutlich, dass auch in Anbetracht der vergleichsweise grossen institutionellen Nähe der Schweiz durch eine detaillierte Analyse bestimmte, bedeutende Unterschiede und Spezifika herausgearbeitet werden können. Unsere Analyse der Schlüsselinstitutionen des Arbeitsmarktes hat gezeigt, dass die grössten Gemeinsamkeiten im Bereich der dualen Berufsausbildung vorliegen. Bezüglich der Kollektivverträge, des Mitbestimmungsrechts sowie arbeitsrechtlicher Rahmenbedingungen hingegen weisen Deutschland und die Schweiz wesentliche Unterschiede auf. Die Schweiz stellt daher einen besonderen Fall dar, der keineswegs mit Deutschland gleichzusetzen ist.

HRM und IB Praktiken, die Konsens, Mitbestimmung und Sensibilität in Kündigungsfragen betreffen, sind in beiden Ländern relativ ähnlich, was eine klare Unterscheidung von country-of-origin und host country Effekten auch aufgrund von Einschränkungen des Forschungsdesigns oftmals schwer oder unmöglich macht. Jedoch zeigt das Beispiel der Schweiz, dass auch andere Institutionen als das deutsche Betriebsverfassungsgesetz zu ähnlichen organisationalen Praktiken führen können.

Verschiedene Praktiken, wie z.B. das Aushandeln von GAV, sind auch stark abhängig von der Belegschaftsstruktur und den Aktivitäten der MNU in der Schweiz sowie von den Gegebenheiten des lokalen Arbeitsmarktes. Unter bestimmten Voraussetzungen, wie sie in der Schweiz vorherrschen, können stabile Beschäftigungsverhältnisse mit ähnlich langen durchschnittlichen Beschäftigungsdauern (OECD 2012a) wie im stark regulierten Umfeld Deutschlands sowie ein grosses Mass an Arbeitsplatzsicherheit auch in einem äusserst flexiblen externen Arbeitsmarkt entstehen.

Wie aus unserer Studie deutlich hervorgeht, kann der flexible Schweizer Arbeitsmarkt bei einer derzeit niedrigen Arbeitslosenquote von 3,1\% (Staatssekretariat für Wirtschaft SECO, 7) starke disziplinierende Wirkung auf die HRM Praktiken der Arbeitgeber entfalten und somit die Stabilität der Beschäftigungsverhältnisse sichern. Hierbei stellt sich die Frage, ob und inwieweit sich diese Wirkung im Falle einer konjunkturellen Eintrübung und steigender Arbeitslosigkeit in ihr Gegenteil verwandeln würde. Es erscheint daher lohnend, die Dynamik des Schweizer Arbeitsmarktes und vor allem seine Wirkung auf HRM Praktiken weiterhin zu beobachten.

\section{Literaturhinweise}

Ackermann, E. (2008): Der SGB und seine Gewerkschaften - Eine Kurzdarstellung mit Hinweisen auf die Geschichte der Gewerkschaften in der Schweiz, unter: http://www.sgb.ch/downloads/Bros chuere_SGB_deutsch.pdf [17.2.2011].

Almond, P. (2011): Re-visiting 'country of origin' effects on HRM in multinational corporations, in: Human Resource Management Journal, Jg. 21, Nr. 3, S. 258-271. 
Almond, P./Edwards, T./Colling, T./Ferner, A./Gunnigle, P. (2005): Unraveling home and host country effects, An investigation of the HR policies of an American multinational in four European countries, in: Industrial Relations, Jg 44, Nr. 2, S. 276-305.

Aubert, G. (2005): L'entreprise et le droit du travail: l'exemple suisse, in: Droit Social (2), S. 147-151, unter: http://archive-ouverte.unige.ch/downloader/vital/pdf/tmp/jch784sirmffpie3nhb tskng70/out.pdf [25.8.2013].

Avery, G./Donnenberg, O./Gick, W./Hilb, M. (1999): Challenges for management development in the German-speaking nations for the twenty-first century, in: Journal of Management Development, Jg.18, Nr. 1, S. 18-31.

Beaumont, P./Cressey, P./Jakobsen, P. (1990): Key industrial relations: West German subsidiaries in Britain, in: Employee Relations 12, Nr. 6, S. 3-7.

Beckert, J. (2003): Economic sociology and embeddedness: how shall we conceptualize economic action? in: Journal of Economic Issues, Jg 37, Nr. 3, S. 769-787.

Bender, C./Grassl, H./Schaal, M. (2007): Der Schweizer Arbeitsmarkt: Sonderfall unter Modernisierungsdruck, in: Eberle, T.S./Imhof, K. (Hrsg.): Sonderfall Schweiz, Zürich, S. 172-187.

Bergmann, A. (1990): Nationale Kultur - Unternehmenskultur, Erkenntnisse aus einer empirischen Untersuchung, in: Die Unternehmung, Jg. 44, Nr. 5, S. 360-370.

Bergmann, A. (1994): Le swiss way of management ou Les évidences cachées des entreprises suisses, Essais, Paris.

Berufsbildungsgesetz (BBiG) (2007).

Betriebsverfassungsgesetz (1972): „Betriebsverfassungsgesetz in der Fassung der Bekanntmachung vom 25. September 2001 (BGBl. I S. 2518), das zuletzt durch Artikel 9 des Gesetzes vom 29. Juli 2009 (BGBl. I S. 2424) geändert worden ist“, BetrVG, unter: http://bundesrecht.juris.de/bundesre cht/betrvg/gesamt.pdf.

Bluhm, K. (2001): Exporting or abandoning the 'German Model'? Labour policies of German manufacturing firms in Central Europe, in: European Journal of Industrial Relations, Jg. 7, Nr. 2, S. 153-173.

Bonvin, J.M. (2007): Corporate social responsibility in a context of permanent restructuring: a case study from the Swiss metalworking sector, in: Corporate Governance: An International Review, Jg. 15, Nr. 1, S. 36-44.

Broussolle, D. (2009): Self-interest, legal commitment and benevolence: the emergence and enforcement of a Swiss labour market institution, in: European Journal of Industrial Relations, Jg. 15, Nr. 3, S. 277-295.

Bundesgesetz über die Berufsbildung (2002), (Berufsbildungsgesetz, BBG).

Chang, S.-I. (2004): Das Konzept des „National Business System“ und der Transfer von HRM-Praktiken multinationaler Unternehmen auf ihre Tochtergesellschaften, Dissertation, Trier.

Chang, S.-I. (2006): Der Transfer von HRM-Praktiken von deutschen multinationalen Unternehmen auf ihre Tochtergesellschaften in Korea, in: Zeitschrift für Personalforschung, Jg. 20, Nr. 3, S. 233-254.

Chevrier, S. (2002): Le solide contre l'ingénieux: malentendus dans la gestion de projets franco-suisses, in: Iribarne, P. d'/Henry, A./Segal, J.-P./Chevrier, S./Globokar, T. (Hrsg.): Cultures et mondialisation. Gérer par-delà les frontières, Collection Points, Jg. 482. Paris, S. 139-163.

Dacin, M.T./Ventresca, M.J./Beal, B.D. (1999): The embeddedness of organizations: dialogue \& directions, in: Journal of Management, Jg. 25, Nr. 3, S. 317-356. 
Davoine, E. (2005): Formation et parcours professionnel des dirigeants d'entreprise en Suisse, in: Dossier de la Revue Économique et Sociale 3, S. 89-99.

Davoine, E./Ravasi, C. (2013): The relative stability of national career patterns in European top management careers in the age of globalisation: a comparative study in France/Germany/Great Britain and Switzerland, in: European Management Journal, Jg. 31, Nr. 2, S. 152-163.

Destatis-Statistisches Bundesamt (2012): Bildungsstand der Bevölkerung 2012, unter: https://www.d estatis.de/DE/Publikationen/Thematisch/BildungForschungKultur/Bildungsstand/BildungsstandBe voelkerung.html [28.5.2013].

Dickmann, M. (2003): Implementing German HRM abroad: desired, feasible, successful?, in: International Journal of Human Resource Management, Jg. 14, Nr. 2, S. 265-283.

DiMaggio, P.J./Powell, W.W. (1983): The iron cage revisited: institutional isomorphism and collective rationality in organizational fields, in: American Sociological Review, Jg. 48, Nr. 2, S. 147-160.

Djelic, M.-L. (1998): Exporting the American model, The post-war transformation of European business, Oxford.

Dörrenbächer, C. (2004): Fleeing or exporting the German model? The internationalization of German multinationals in the 1990s, in: Competition \& Change, Jg. 8, Nr. 4, S. 443-456.

Dörrenbächer, C./Gammelgaard, J. (2011): Subsidiary power in multinational corporations: the subtle role of micro-political bargaining power, in: Critical Perspectives on International Business, Jg. 7, Nr. 1, S. 30-47.

Edwards, T./Almond, P./Clark, I./Colling, T./Ferner, A. (2005): Reverse diffusion in US multinationals: barriers from the American business system, in: Journal of Management Studies, Jg. 42, Nr. 6, S. 1261-1286.

Edwards, T./Colling, T./Ferner, A. (2007): Conceptual approaches to the transfer of employment practices in multinational companies: an integrated approach, in: Human Resource Management Journal, Jg. 17, Nr. 3, S. 201-217.

Edwards, T./Ferner, A. (2002): The renewed 'American Challenge': a review of employment practice in US multinationals, in: Industrial Relations Journal, Jg. 33, Nr. 2, S. 94-111.

Edwards, T./Rees, C. (2006): Chapter 4: International strategy and structure in multinational companies, in: Edwards, T./Rees, C. (Hrsg.): International human resource management: Globalization, national systems and multinational companies, Harlow, S. 66-87.

Eidgenössisches Departement für Wirtschaft, B. u. F. (2013): Fakten und Zahlen - Berufsbildung in der Schweiz 2013, unter: http://www.sbfi.admin.ch/dokumentation/00335/00400/index.html?lan $\mathrm{g}=\mathrm{de}$ [28.5.2013].

Eisenhardt, K.M. (1989): Building theories from case study research, in: The Academy of Management Review, Jg. 14, Nr. 4, S. 532-550.

Eisenhardt, K.M./Graebner, M.E. (2007): Theory building from cases: opportunities and challenges, in: Academy of Management Journal, Jg. 50, Nr. 1, S. 25-32.

Erten, C./Strunk, G./Gonzalez, J.-C./Hilb, M. (2004): Austria and Switzerland: small countries with large differences, in: Brewster, C./Mayrhofer, W./Morley, M. (Hrsg.): Human resource management in Europe. Evidence of convergence?, S. 95-122.

Faulkner, D./Pitkethly, R./Child, J. (2002): International mergers and acquisitions in the UK 1985-94: a comparison of national HRM practices, in: The International Journal of Human Resource Management, Jg. 13, Nr. 1, S. 106-122. 
Ferner, A. (1994): Multinational companies and human resource management: an overview of research issues, in: Human Resource Management Journal, Jg. 4, Nr. 2, S. 79-101.

Ferner, A. (1997): Country of origin effects and HRM in multinational companies, in: Human Resource Management Journal, Jg. 7, Nr. 1, S. 19-37.

Ferner, A. (2001): The embeddedness of US multinational companies in the US business system, Implications for HR/IR, Leicester.

Ferner, A./Almond, P./Clark, I./Colling, T./Edwards, T./Holden, L./Müller-Camen, M. (2004): The dynamics of central control and subsidiary autonomy in the management of human resources: case-study evidence from US MNCs in the UK, in: Organization Studies, Jg. 25, Nr. 3, S. 363-391.

Ferner, A./Edwards, T./Tempel, A. (2012): Power, institutions and the cross-national transfer of employment practices in multinationals, in: Human Relations, Jg. 65, Nr. 2, S. 163-187.

Ferner, A./Quintanilla, J. (1998): Multinationals, national business systems and HRM: the enduring influence of national identity or a process of 'Anglo-Saxonization', in: International Journal of Human Resource Management, Jg. 9, Nr. 4, S. 710-731.

Ferner, A./Quintanilla, J./Varul, M.Z. (2001): Country-of-origin effects, host-country effects, and the management of HR in multinationals: German companies in Britain and Spain, in: Journal of World Business, Jg. 36, Nr. 2, S. 107-127.

Ferner, A./Varul, M.Z. (2000a): 'Vanguard' subsidiaries and the diffusion of new practices: a case study of German multinationals, in: British Journal of Industrial Relations, Jg. 38, Nr. 1, S. 115-140.

Ferner, A./Varul, M.Z. (2000b): Internationalisation and the personnel function in German multinationals, in: Human Resource Management Journal, Jg. 10, Nr. 3, S. 79-96.

Fischer, A. (2003): Vetospieler und die Durchsetzbarkeit von Side-Payments, Der schweizerische innenpolitische Entscheidungsprozess um flankierende Massnahmen zur Personenfreizügigkeit mit der Europäischen Union, in: Swiss Political Science Review, Jg. 9, Nr. 2, S. 27-58.

Flanagan, R.J. (1999): Macroeconomic performance and collective bargaining: an international perspective, in: Journal of Economic Literature, Jg. 37, Nr. 3, S. 1150-1175.

Freitag, M. (2004): Schweizer Welten des Sozialkapitals, Empirische Untersuchungen zum sozialen Leben in Regionen und Kantonen, in: Swiss Political Science Review, Jg. 10, Nr. 2, S. 87-118.

Friel, D. (2005): Transferring a lean production concept from Germany to the United States: the impact of labor laws and training systems, in: Academy of Management Executive, Jg. 19, Nr. 2, S. 50-58.

Fumagalli-Senn, M. (2009): Erhebung der Gesamtarbeitsverträge in der Schweiz 2007, Statistik der Schweiz. Fachbereich 3, Arbeit und Erwerb, Neuchâtel.

Giardini, A./Kabst, R./Müller-Camen, M. (2005): HRM in the German business system. A review, in: Management Revue, Jg. 16, Nr. 1, S. 63-80.

Gibbert, M./Ruigrok, W./Wicki, B. (2008): What passes as a rigorous case study? in: Strategic Management Journal, Jg. 29, Nr. 13, S. 1465-1474.

Gonon, P. (1999): Neue Reformbestrebungen im beruflichen Bildungswesen in der Schweiz, in: Berufsbildung, Jg. 17, S. 48-54.

Gonon, P. (2005): Challenges in the Swiss vocational education and training system, unter: http://w ww.bwpat.de/7eu/gonon_ch_bwpat7.pdf [19.2.2011].

Granovetter, M. (1985): Economic action and social structure: the problem of embeddedness, in: The American Journal of Sociology, Jg. 91, Nr. 3, S. 481-510. 
Guertzgen, N. (2009): Rent-sharing and collective bargaining coverage: evidence from linked employer-employee data, in: Scandinavian Journal of Economics, Jg. 111, Nr. 2, S. 323-349.

Guest, D.E./Hoque, K. (1996): National ownership and HR practices in UK greenfield sites, in: Human Resource Management Journal, Jg. 6, Nr. 4, S. 50-74.

Hall, P.A./Gingerich, D.W. (2004): Varieties of capitalism and institutional complementarities in the macroeconomy. An empirical analysis, unter: http://www.gbv.de/dms/sub-hamburg/470362316. pdf.

Hall, P.A./Gingerich, D.W. (2009): Varieties of capitalism and institutional complementarities in the political economy: an empirical analysis, in: British Journal of Political Science, Jg. 39, Nr. 03, S. 449-482.

Hanhart, S./Bossio, S. (1998): Costs and benefits of dual apprenticeship: lessons from the Swiss system, in: International Labour Review, Jg. 137, Nr. 4, S. 483-500.

Hartley, J. (2004): Case study research, in: Cassell, C./Symon, G. (Hrsg.): Essential guide to qualitative methods in organizational research, London/Thousand Oaks, S. 323-333.

Hayter, S./Fashoyin, T./Kochan, T.A. (2011): Review essay: collective bargaining for the 21st century, in: Journal of Industrial Relations, Jg. 53, Nr. 2, S. 225-247.

Hoeckel, K. (2008): Costs and benefits in vocational education and training, unter: http://www.oecd .org/edu/skills-beyond-school/41538706.pdf [21.8.2013].

Hofstede, G. (2001): Culture's consequences, Comparing values, behaviors, institutions and organizations across nations, Thousand Oaks.

Kenworthy, L. (2003): Quantitative indicators of corporatism, in: International Journal of Sociology, Jg. 33, Nr. 3, S. 10-44.

King, N. (2004a): Using interviews in qualitative research, in: Cassell, C./Symon, G. (Hrsg.): Essential guide to qualitative methods in organizational research, London/Thousand Oaks, S. 11-22.

King, N. (2004b): Using templates in the thematic analysis of text, in: Cassell, C./Symon, G. (Hrsg.): Essential guide to qualitative methods in organizational research. London/Thousand Oaks, S. 256-270.

Kostova, T./Roth, K./Dacin, M.T. (2008): Institutional theory in the study of multinational corporations: a critique and new directions, in: Academy of Management Review, Jg, 33, Nr. 4, S. 994-1006.

Laurent, A. (1985): The cultural diversity of Western conceptions of management, in: Joynt, P./ Warner, M. (Hrsg.): Managing in different cultures, Oslo/Irvington-on-Hudson, S. 41-56.

Lesch, H. (2003): Der Arbeitskampf als Instrument tarifpolitischer Konfliktbewältigung, in: Aus Politik und Zeitgeschichte, Jg. 53, Nr. 47/48, S. 30-38, unter: http://www.bpb.de/files/QC9PVM.pd $\mathrm{f}[14.2 .2011]$.

Mahon, P. (2000): L'évolution récente du droit du travail en Suisse - une appréciation critique, in: Aspects de la Sécurité Sociale, Nr.3, S. 9-26, unter: http://www.feas.ch/pdf_revue/2000_03.pdf [25.8.2011].

Marginson, P./Buitendam, A./Deutschmann, C./Perulli, P. (1993): The emergence of the Euro-company: towards a European industrial relations?, in: Industrial Relations Journal, Jg. 24, Nr. 3, S. 182-190.

Marginson, P./Edwards, P.K./Edwards, T./Ferner, A./Tregaskis, O. (2010): Employee representation and consultative voice in multinational companies operating in Britain, in: British Journal of Industrial Relations, Jg. 48, Nr.1, S. 151-180. 
Marschan-Piekkari, R./Reis, C. (2004): Language and languages in cross-cultural interviewing, in: Marschan-Piekkari, R./Welch, C. (Hrsg.): Handbook of qualitative research methods for international business, Cheltenham/Northampton, S. 224-243.

Meardi, G. (2007): More voice after more exit? Unstable industrial relations in Central Eastern Europe, in: Industrial Relations Journal, Jg. 38, Nr. 6, S. 503-523.

Meardi, G./Marginson, P./Fichter, M./Frybes, M./Stanojević, M. (2009): Varieties of multinationals, Adapting employment practices in Central Eastern Europe, in: Industrial Relations, Jg. 48, Nr. 3, S. 489-511.

Meunier, M. (2007): Analyse économique de la production éducationnelle le cas de la Suisse Thèse de doctorat, Genève.

Meyer, J.W./Rowan, B. (1977): Institutionalized organizations, Formal structure as myth and ceremony, in: The American Journal of Sociology, Jg. 83, Nr. 2, S. 340-363.

Mitwirkungsgesetz (1993): Bundesgesetz vom 17. Dezember 1993 über die Information und Mitsprache der Arbeitnehmerinnen und Arbeitnehmer in den Betrieben (Mitwirkungsgesetz), unter: http://www.admin.ch/ch/d/sr/c822_14.html [14.2.2011].

Morley, M./Brewster, C./Gunnigle, P./Mayrhofer, W. (2000): Evaluating change in European industrial relations: research evidence on trends at organisational level, in: Brewster, C./Mayrhofer, W./Morley, M. (Hrsg.): New challenges for European human resource management, New York, S. 199-221.

Müblemann, S./Wolter, S.C. (2007): Lehrlingsausbildung lohnt sich, in: Die Volkswirtschaft, Nr. 10, S. 44-47, unter: http://www.dievolkswirtschaft.ch/editions/200710/pdf/Muehlemann.pdf [20.8.2013].

Müller, F.U. (1994): Societal effect, organizational effect and globalization, in: Organization Studies, Jg. 15, Nr. 3, S. 407-428.

Müller, M. (1999): Unitarism, pluralism, and human resource management in Germany, in: Management International Review, Jg. 39, Nr. 3, S. 125-144.

Naville, M., et al. (2007): Multinational companies on the move: how Switzerland will win the battle!, unter: http://www.osec.ch/internet/osec/en/home/invest/worldwide/publications.-RelatedBox Slot-52403-ItemList-56531-File.File.pdf/pub_mult_companies_on_the_move.pdf [17.2.2011].

Nikolai, R. (2005): Die Arbeitsmarkt-und Beschäftigungspolitik in der Schweiz, Die Schweiz als Erfolgsmodell? Auszug aus: Debatte: Wirtschaft und Politik in der Schweiz, in: Swiss Political Science Review, Jg. 11, Nr. 3, S. 193-197.

OECD (2010): Learning for jobs, Synthesis report of the OECD reviews of vocational education and training, OECD reviews of vocational education and training.

OECD (2012a): OECD.Stat Extracts - employment by job tenure intervals - average tenure, unter: http://stats.oecd.org/Index.aspx?QueryId=9591\# [26.5.2012].

OECD (2012b): OECD.Stat Extracts - trade union density, unter: http://stats.oecd.org/Index.aspx? DataSetCode=UN_DEN [25.5.2012].

Pudelko, M./Harzing, A.W. (2007): Country-of-origin, localization, or dominance effect? An empirical investigation of HRM practices in foreign subsidiaries, in: Human Resource Management, Jg. 46, Nr. 4, S. 535-559.

Royle, T. (2004): Employment practices of multinationals in the Spanish and German quick-food sectors: low-road convergence? in: European Journal of Industrial Relations, Jg. 10, Nr. 1, S. 51-71. 
Scharnhorst, U. (2013): Joint governance and financing of VET in Switzerland: advantages and challenges, Apprenticeship: governance modes and financing approaches. Cedefop workshop, Thessaloniki, 20 - 21 May 2013, unter: http://www.cedefop.europa.eu/EN/Files/07_Joint_governance _and_financing_of_VET_in_Switzerland_-_advantages_and_challenges_Ursula_Scharnhorst.pdf [21.8.2013].

Scheuer, S. (2006): A novel calculus? Institutional change, globalization and industrial conflict in Europe, in: European Journal of Industrial Relations, Jg. 12, Nr. 2, S. 143-164.

Schmid, H. (2001): Industrial relations in changing times, in: International Journal of Applied Economics \& Econometrics, Jg. 9, Nr. 4, S. 451-477.

Schmitt, M. (2003): Deregulation of the German industrial relations system via foreign direct investment: are the subsidiaries of Anglo-Saxon MNCs a threat for the institutions of industrial democracy in Germany?, in: Economic and Industrial Democracy, Jg. 24, Nr. 3, S. 349-377.

Schneider, M.R./Paunescu, M. (2012): Changing varieties of capitalism and revealed comparative advantages from 1990 to 2005: a test of the Hall and Soskice claims, in: Socio-Economic Review, Jg. 10, Nr. 4, S. 731-753.

Schwab, K./Sala-i-Martin, X. (2012): The global competitiveness report 2012-2013, Full Data Edition, The Global Competitiveness Report, Geneva.

Sinkovics, R.R./Penz, E./Ghauri, P.N. (2008): Enhancing the trustworthiness of qualitative research in international business, in: Management International Review, Jg. 48, Nr. 6, S. 689-714.

Smith, C./Meiksins, P. (1995): System, society and dominance effects in cross-national organisational analysis, in: Work, Employment \& Society, Jg. 9, Nr. 2, S. 241-267.

Soskice, D.W. (1990): Wage determination, The changing role of institutions in advanced industrialized countries, in: Oxford Review of Economic Policy, Jg. 6, Nr. 4, S. 36-61.

Soskice, D.W. (1999): Globalisierung und institutionelle Divergenz: Die USA und Deutschland im Vergleich, in: Geschichte und Gesellschaft, Jg. 25, Nr. 2, S. 201-225.

Sousa-Poza, A. (2004): Job stability and job security, A comparative perspective on Switzerland's experience in the 1990s, in: European Journal of Industrial Relations, Jg. 10, Nr. 1, S. 31-49.

Staatssekretariat für Wirtschaft SECO: Die Lage auf dem Arbeitsmarkt im April 2013, unter: http:// www.seco.admin.ch/themen/00374/00384/ [30.5.2013].

Streeck, W. (1987): The uncertainties of management in the management of uncertainty, in: International Journal of Political Economy, Jg. 17, Nr. 3, S. 57-87.

Tempel, A. (2001): The cross-national transfer of human resource management practices in German and British multinational companies, München.

Tempel, A./Edwards, T./Ferner, A./Müller-Camen, M./Wächter, H. (2006): Subsidiary responses to institutional duality: collective representation practices of US multinationals in Britain and Germany, in: Human Relations, Jg. 59, Nr. 11, S. 1543-1570.

Teuber, S./Backes-Gellner, U. (2013): How do companies adjust their organization to national institutions: evidence from matched-pair engineering companies, Swiss Leading House Working Paper (82), S. 1-31, unter: http://ideas.repec.org/p/iso/educat/0082.html [23.8.2013].

Tijdens, K./van Klaveren, M. (2007): Collective bargaining coverage, Wageindicator support for trade union bargaining in Europe (WIBAR) supported by the European commission in its industrial relations and social dialogue program, unter: http://www.wageindicator.org/documents/pub licationslist/WIBARCBC [28.2.2011].

Tixier, M. (1994): Management and communication styles in Europe: can they be compared and matched? in: Employee Relations, Jg. 16, Nr. 1, S. 8-26. 
Tüselmann, H.-J., et al. (2001): German multinationals in the UK and the German model of labour relations: diffusion and reverse diffusion effects, unter: http://www.ribm.mmu.ac.uk/wps/papers/ 01-06.pdf [18.2.2011].

Tüselmann, H.-J./McDonald, F./Heise, A. (2003): Employee relations in German multinationals in an Anglo-Saxon setting, Toward a Germanic version of the Anglo-Saxon approach?, in: European Journal of Industrial Relations, Jg. 9, Nr. 3, S. 327-349.

Tüselmann, H.-J./McDonald, F./Thorpe, R. (2006): The emerging approach to employee relations in German overseas affiliates: a role model for international operation?, in: Journal of World Business, Jg. 41, Nr. 1, S. 66-80.

Venn, D. (2009): Legislation, collective bargaining and enforcement, Updating the OECD employment protection indicators, unter: http://www.oecd.org/dataoecd/36/9/43116624.pdf.

Visser, J. (2006): Union membership statistics in 24 countries, in: Monthly Labor Review, Jg. 129, Nr. 1, S. 38-49 [14.2.2011].

Wächter, H., et al. (2003): The 'country-of-origin-effect' in the cross-national management of human resources, Results and case study evidence of research on American multinational companies in Germany, 1. Auflage, München.

Wächter, H. (2004): Einleitung und Überblick, in: Wächter, H./Peters, R. (Hrsg.): Personalpolitik amerikanischer Unternehmen in Europa, Trierer Beiträge zum Diversity Management, Band 4, München, S. 3-6.

Wächter, H./Müller-Camen, M. (2002): Co-determination and strategic integration in German firms, in: Human Resource Management Journal, Jg. 12, Nr. 3, S. 76-87.

Wächter, H./Peters, R. (2004a): Die Europäisierung der Personalpolitik? in: Wächter, H./Peters, R. (Hrsg.): Personalpolitik amerikanischer Unternehmen in Europa, Trierer Beiträge zum Diversity Management, Band 4, München, S. 181-188.

Wächter, H./Peters, R. (Hrsg.) (2004b): Personalpolitik amerikanischer Unternehmen in Europa, Trierer Beiträge zum Diversity Management, Band 4, München.

Wächter, H./Stengelhofen, T. (1992): Human resource management in a unified Germany, in: Employee Relations, Jg. 14, Nr. 4, S. 21-37.

Warner, M./Campbell, A. (1993): German management, in: Hickson, D.J. (Hrsg.): German management: Society, culture and organization in twelve nations, Berlin/New York, S. 89-108.

Weibler, J./Wunderer, R. (2007): Leadership and culture in Switzerland, Theoretical and empirical findings, in: Chbokar, J.S./Brodbeck, F.C./House, R.J. (Hrsg.): Culture and leadership across the world, The GLOBE book of in-depth studies of 25 societies, Mahwah, N.J., S. 251-295.

Wever, K.S. (1995): Human resource management and organizational strategies in German and USowned companies, in: The International Journal of Human Resource Management, Jg. 6, Nr. 3, S. 606-625.

Williams, K./Geppert, M. (2006): Employment relations as a resource in the socio-political construction of transnational social spaces by multinational companies, Evidence from German and British subsidiaries, in: Geppert, M./Mayer, M. (Hrsg.): Global, national and local practices in multinational companies, Basingstoke, S. 38-60.

Winterton, J. (2007): Building social dialogue over training and learning: European and national developments, in: European Journal of Industrial Relations, Jg. 13, Nr. 3, S. 281-300.

Wolter, S.C. (2005): Ländervergleich: Kosten der beruflichen Grundbildung. Schweizer Lehrlinge sind billiger als deutsche, in: Panorama, Nr. 2, S. 20-21. 
Wolter, S.C./Schweri, J. (2003): Kosten und Nutzen der Lehrlingsausbildung aus der Sicht Schweizer Betriebe, Zusammenfassung und Einzelaspekte der Studienergebnisse, unter: www.sbfi.admin.ch/ dokumentation/00335/00400/index.html?lang=de\&download=NHzLpZeg7t, lnp6I0N-

TU04212Z6ln1acy4Zn4Z2qZpnO2Yuq2Z6gpJCDdXt7fmym162epYbg2c_JjKbNoKSn6A-[21.8.2013].

Yin, R.K. (2009): Case study research, Design and methods, 4. Auflage, Los Angeles.

Oliver Schröter, M.A., ist Doktorand und Assistent am Lehrstuhl für Personal und Organisation der Universität Freiburg/Schweiz.

Anschrift: Université de Fribourg, Chaire Ressources Humaines et Organisation, Boulevard de Pérolles 90, CH-1700 Fribourg, Tel.: +41 (0)26 $300 \quad 82$ 42, Fax: +41 (0)26/300-96-33, E-Mail: oliverchristian.schroeter@unifr.ch

Eric Davoine, Dr., ist Professor für Personal und Organisation an der Universität Freiburg/ Schweiz.

Anschrift: Université de Fribourg, Chaire Ressources Humaines et Organisation, Boulevard de Pérolles 90, CH-1700 Fribourg, Tel.: +41 (0)26 30082 42, Fax: +41 (0)26/300-96-33, E-Mail: eric.davoine@unifr.ch 